\title{
The Local Tully-Fisher Relation for Dwarf Galaxies
}

\author{
Igor D. Karachentsev, Elena I. Kaisina, Olga G. Kashibadze (Nasonova) \\ Special Astrophysical Observatory, Nizhniy Arkhyz, Karachai-Cherkessia 369167, Russia
}

\begin{abstract}
We study different incarnations of the Tully-Fisher (TF) relation for the Local Volume (LV) galaxies taken from Updated Nearby Galaxy Catalog. The UNGC sample contains 656 galaxies with $W_{50}$ HI-line-width estimates, mostly belonging to low mass dwarfs. Of them, 296 objects have distances measured with accuracy better than $10 \%$. For the sample of $331 \mathrm{LV}$ galaxies having baryonic masses $\log M_{b a r}>5.8 \log M_{\odot}$ we obtain a relation $\log M_{\text {bar }}=2.49 \log W_{50}+3.97$ with observed scatter of 0.38 dex. The largest factors affecting the scatter are observational errors in $K$-band magnitudes and $W_{50}$ line widths for the tiny dwarfs, as well as uncertainty of their inclinations. We find that accounting for the surface brightness of the LV galaxies, or their gas fraction, or specific star formation rate, or the isolation index do not reduce essentially the observed scatter on the baryonic TF-diagram. We also notice that a sample of $71 \mathrm{dSph}$ satellites of the Milky Way and M31 with known stellar velocity dispersion $\sigma^{*}$ tends to follow nearly the same bTF relation, having slightly lower masses than that of late-type dwarfs.
\end{abstract}

Subject headings: galaxies: dwarf - galaxies: dark matter - galaxies: kinematics and dynamics

\section{Introduction}

Since the first wholesale observations of galaxies in the HI $21 \mathrm{~cm}$ line, some tight correlations between different integral properties of spiral galaxies were exposed (Roberts 1969, Balkowski et al. 1974). Later Tully \& Fisher (1977) noticed the clear power-law correlation between HI $21 \mathrm{~cm}$ line width and optical luminosity of galaxies, and suggested to use this dependence to measure distances of galaxies. Since then, more than a thousand articles have appeared, addressing to the Tully-Fisher $(=\mathrm{TF})$ relation in different bands of optical and infrared wavelength with different indicators of inner rotation amplitudes. Tully \& Pierce (2000) have calibrated the TF relation in $B, R, I$, and $K$ bands, mentioning that the slope of the TF relation grows systematically with wavelength. According to these authors, scatter on the diagram is small for massive disk galaxies, allowing to measure distances of spiral galaxies with an accuracy of about $20 \%$. A linear relation between $\mathrm{HI}$ line width $W_{50}$ measured at the $50 \%$ level of the maximum and standard optical diameter for flat edge-on galaxies (Karachentsev 1989; Karachentsev et al. 1999) is a particular instance of the TF 
relation. For Sc-Sd galaxies with apparent axis ratio $a / b>7$, this method also yields the distance accuracy of $\sim 20 \%$.

Later, McGaugh and coauthors noted that TF relation connecting mass of a dark halo and baryonic mass of a galaxy should additionally account for mass of a gas component (McGaugh 2005; 2012, McGaugh et al. 2010). This correction turned out to be mostly significant for dwarf irregular galaxies evolving slower than spirals, with extant primordial gas presumably prevailing over star component. This version of Tully-Fisher diagram is known as the baryonic TF (= bTF) relation.

The observed scatter in the TF diagram keeps steadily growing from massive spirals to dwarf galaxies. The main reason of the scatter is the growth of observational uncertainties in apparent magnitudes and line widths $\log W_{50}$ increasing towards dwarfs. Another reason may be the shallow potential well of a dwarf galaxy where gas can easily escape due to some external factors. One more reason for the scatter may be the irregular shape of dwarfs making it difficult to determine an inclination of rotation axis to the line of sight. Moreover, HI imaging study of low-mass dIr galaxies performed with the Giant Metrewave Radio Telescope (GMRT) showed that a direction of HI major axis for some tiny dwarfs does not always coincide with the direction of their optical major axis (Begum et al. 2008a). It should be noted that the neutral hydrogen layers of many galaxies may be warped: the inclination may significantly change in the outer parts (e.g., Garcia-Ruiz \& Sancisi 2002). Optical inclinations are often inappropriate for warped HI discs. These circumstances make rather uncertain the correction of rotation amplitude for dwarf galaxy inclination unless spatially resolved HI observations are available. Finally, dwarf galaxies usually have slowly rising rotation curves and reach the $V_{\text {flat }}$ value in hardly detected peripheric regions. As a result, their $V_{m}=W_{50} /(2 \times \sin i)$ corresponds to only a part of the full rotation amplitude (Swaters et al. 2009). It should be also noted that bursts of star formation in a dwarf galaxy affect its integral luminosity more significantly than in a massive galaxy, causing one more source of scatter towards dwarf sector of the TF diagram.

The main goal of our paper is to improve a calibration of the TF relation on its low-mass

end, using the local dwarf galaxies with accurately measured distances, that allows to up-date the distances of many remaining nearby dwarfs with already known HI line widths.

\section{The Local Volume sample.}

For obvious reasons, the most representative and homogeneous sample of dwarf galaxies can be obtained examining the closest volume of the Universe. Kraan-Korteweg \& Tammann (1979) have made the first attempt to compile such a sample. Their list included 179 galaxies with radial velocities $V_{L G}<500 \mathrm{~km} / \mathrm{s}$ relative to the centroid of the Local Group. Due to the systematic sky surveys in the optical range and HI $21 \mathrm{~cm}$ line, the number of nearby galaxies with similar velocities grew rapidly. 25 years later, the Catalog of Neighboring Galaxies (=CNG, Karachentsev 
et al. 2004) amounted to 450 galaxies in the volume limited to depth of $10 \mathrm{Mpc}$. The refined and up-to-date version of this catalog, Updated Nearby Galaxy Catalog (=UNGC, Karachentsev et al. 2013) contains 869 galaxies with individual distance estimates $D<11 \mathrm{Mpc}$ or radial velocities $V_{L G}<600 \mathrm{~km} / \mathrm{s}$. More than $85 \%$ of this sample are dwarf galaxies with luminosities less than those of Magellanic Clouds. Various observational data on these objects including their images are compiled in the Catalog \& Atlas of the Local Volume Galaxies (Kaisina et al. 2012, http://www.sao.ru/lv/lvgdb/). The database is still being enlarged with new, mainly dwarf, galaxies and contains (as on September 2016) already 1049 objects distributed all over the whole sky. We use this sample below to construct the TF diagram in its different incarnations.

The data on HI fluxes of galaxies, $F(H I)$, expressed in HI-magnitudes $m_{21}=17.4-2.5 \log F(H I)$ according to Paturel et al. (1997), as well as HI $21 \mathrm{~cm}$ line widths, $W_{50}$, originate mainly from HI sky surveys: the HI Parkes All Sky Survey (=HIPASS; Koribalsky et al. 2004; Meyer et al. 2004; Wong et al. 2006; Staveley-Smith et al. 2016), Arecibo Legacy Fast ALFA Survey (=ALFALFA; Giovanelli et al. 2005; Haynes et al. 2011), Westerbork Survey (Kovaĉ et al. 2009) and special observations of selected dwarf galaxies (Huchtmeier et al. 2000, 2001, 2003; Begum et al. 2008a). The individual references to these and other HI data are represented in the Local Volume $(=\mathrm{LV})$ database (http://www.sao.ru/lv/lvgdb/).

Table 1 presents numbers of the LV galaxies with distances measured via different methods. The first column corresponds to the total UNGC sample updated with recent observations, the second column refers to UNGC objects detected in the HI line. The first row shows the number of galaxies whose distances are measured with errors less than $10 \%$ : from the tip of red giant branch (TRGB), Cepheid luminosity (Cep), Supernovae (SN), RR Lyra variables (RR), color-magnitude diagram (CMD), and the horizontal branch (HB). The second row gives the number of galaxies with distance estimates obtained from surface brightness fluctuations (SBF). The accuracy of this method, according to Tonry et al. (2001), is also about 10\%, but this method is suitable for early-type galaxies with low amount of dust and gas, leading to the risk that the $W_{50}$ value for them can differ systematically from that of late-type galaxies of the same luminosity. The third row of the table refers to galaxies with distances determined from their membership (mem) in groups, where other (usually brighter) members have individual distance estimates. According to recent TRGB-distance measurements for galaxies with earlier fixed membership, the mean distance error for this category of galaxies is estimated by us as $17 \%$. Galaxies with distances determined from the ordinary Tully-Fisher relation (TF) or its baryonic version (bTF) are denoted in the fourth row. The fifth row corresponds to galaxies with distance estimates collected from less reliable methods: luminosity of the brightest stars (bs), planetary nebula luminosity function (PN) or from apparent texture of an object (txt). The sixth row shows the number of galaxies with kinematic distance estimates obtained from their radial velocities where the Hubble parameter is set to $H_{0}=73 \mathrm{~km} \mathrm{~s}^{-1} \mathrm{Mpc}^{-1}$ and the virgocentric velocities are factored (h') or not (h). Finally, the total number of the LV galaxies with different distance estimates is shown in the last row.

As seen, our LV sample contains a total of 656 galaxies with estimates of $W_{50}$ and $m_{21}$. Almost 
half of them have distances measured with an accuracy of $5-10 \%$. This circumstance is particularly significant for nearby galaxies, because their small radial velocities can't be a robust indicator of distance being affected by peculiar motions.

A literature review shows that authors often preselect galaxies by some specific properties complicating the interpretation of TF diagram. For instance, Papastergis et al. (2016) in their study of the baryonic TF relation for heavily gas-dominated ALFALFA objects, selected only galaxies with apparent axial ratio less than 0.25 , omitting a huge number of low mass galaxies which are gasrich but intrinsically thick. Sometimes authors do not completely specify their selection criteria or neglect galaxies deviating significantly from the $\mathrm{TF}$ regression line. It results in artificially reducing scatter in the TF relation. Below, we look toward keeping in our sample as many objects as possible, eliminating only face-on disks, or galaxies located in the Zone of Avoidance, or those with ambiguous distance estimates. In particular, we did not omit from our analisys a case of nearby bright galaxy M82 having accurate photometric data, the reliable estimate of the distance, $W_{50}$ width and inclination $i$, albeit this is a known starbust galaxy involved in a strong interaction with M81 impressively visible in HI (Yun et al. 1994).

\section{Intrinsic axial ratio and inclination corrections.}

The distribution of apparent axis ratios for the LV galaxies of different morphological types is shown in Figure 1. The galaxies are marked by small circles. Many of them overlap with each other. The large circles and vertical rectangles indicate the mean values and the standard deviations for each type in de Vaucouleurs scale. The left side of the diagram $(T<1)$ contains mostly HI undetected objects. In general, the intrinsic axial ratio is larger for lenticular and irregular galaxies than for spirals, and among the spirals is smaller for the late types than for the early ones. The minimum flattening corresponds to the Sd galaxies $(\mathrm{T}=7)$, although a thin edge-on galaxy would be classified as $\mathrm{Sd}$ instead of $\mathrm{Sc}$ or $\mathrm{Sm}$ since one cannot appreciate the degree of structure in the pattern at $i \simeq 90 \mathrm{deg}$.

As it was already mentioned, uncertainties in correction for inclination of dwarf galaxies may

Table 1: Numbers of the Local Volume galaxies with distances measured by different methods.

\begin{tabular}{lcc}
\hline Method & $N_{\text {all }}$ & $N_{H I}$ \\
\hline TRGB, Cep, SN, RR, CMD, HB & 459 & 296 \\
SBF & 18 & 9 \\
mem & 269 & 85 \\
TF, bTF & 189 & 188 \\
bs, PN, txt & 38 & 24 \\
h, h' & 76 & 54 \\
\hline All methods & 1049 & 656 \\
\hline
\end{tabular}


cause scatter in the TF diagram. For oblate ellipsoids, an inclination of rotation axis of a galaxy to the line of sight is defined as

$$
\sin ^{2} i=\left[1-q^{2}\right] /\left[1-q_{0}^{2}\right],
$$

where $q=b / a$ is the apparent axial ratio. In case of CNG sample, the intrinsic flattenings $q_{0}=$ $(b / a)_{0}$ were taken equal to 0.07 for morphological types $T=6$ or $7,0.12$ for types 5 or $8,0.18$ for types 4 or 9 and 0.20 for all other morphological types, according to the Second Reference Catalog (RC2) by de Vaucouleurs et al. (1976). This relation between the intrinsic axial ratio and de Vaucouleurs morphological type is represented in Fig. 1 by a dotted line with open circles. Analyzing the apparent axial ratio statistics for dwarf galaxies taken from the UNGC, Roychowdhury et al. (2013) confirmed the known fact that disks of dwarf galaxies are much thicker than it was assumed in RC2. These authors obtained the mean intrinsic flattening of 0.57. Just the same value was found by Sanchez-Janssen et al. (2016) for dwarf population of the Virgo cluster. The authors of both papers noted that the apparent axial ratio statistics for dwarf galaxies is better described by a model of oblate triaxial spheroid with axes ratio $1.00: 0.94: 0.57$. Also, Roychowdhury et al. (2010) derived approximately the same axial ratio for HI images of dIr galaxies based on FIGGS data from GMRT (Begum et al. 2008a).

Paturel et al. (1997) suggested a refined set of $q_{0}$ values depending on morphological type $T$, which was used in UNGC to determine the inclination $i$ from relation (1):

$$
\begin{gathered}
\log \left(q_{0}\right)=-0.43-0.053 \times T \text { for } T \leq 8 \\
\log \left(q_{0}\right)=-0.38 \text { for } T=9,10
\end{gathered}
$$

The behaviour of intrinsic flattenings versus morphological type in this case is depicted by the dashed line with grey circles in the Fig. 1. The recipe of Paturel et al. (1997) for $q_{0}$ seems to be significantly more realistic than the previous scheme used in RC2. Yet, the condition (2) presumably underestimates the actual thickness of the disks of dwarf galaxies, resulting in their inclination $i$ estimates. Here we should point out a big leap in the condition (2) passing from morphological type $T=8$ to 9 . A typical scatter in morphological classification of $\Delta T= \pm 1$ can lead to a significant error in galaxy inclination.

Yuan \& Zhu (2004) analyzed a sample of 14988 disk galaxies taken from LEDA database and derived for them the relation

$$
\begin{gathered}
\log \left(q_{0}\right)=-0.580-0.067 \times T \text { for } T \leq 7 \\
\log \left(q_{0}\right)=-2.309+0.185 \times T \quad \text { for } T=8,9,10 .
\end{gathered}
$$

This distribution is shown in the Figure 1 by the solid line with black circles. It looks as intermedian between those adopted in CNG and UNGC samples for most morphological types.

There is another way to evaluate the intrinsic axial ratio using stellar mass instead of optical morphology. Sanchez-Janssen et al. (2010) investigated the role of stellar mass in shaping the 
intrinsic thickness of dwarf systems. They found that the intrinsic axial ratio varies with stellar mass in a parabolic fashion having the minimum value near the stellar mass $M^{*}=2 \times 10^{9} M_{\odot}$. In our LV sample the distribution of all 1049 galaxies according to their total luminosity in K-band, $L_{K}$, and morphological type is shown in Figure 2. Assuming an approximate ratio $M^{*} / L_{K} \simeq 1 M_{\odot} / L_{\odot}$ (Bell et al. 2003) this diagram can be transformed into the $M^{*}$ vs. T distribution. The mean values of $L_{K}$ and standard deviations are marked, like in Fig. 1, by large circles and rectangles. The shape of diagram resembles parabola with the maximum near Sb-type and a scatter increasing towards both the sides.

The number distribution of all LV galaxies with their K-band luminosity is illustrated by Figure 3. Among them, the 656 HI-detected galaxies are marked by dark color. As one can see, a fraction of the LV galaxies with the HI detections decreases systematically from bright to faint objects. More than $99 \%$ of the HI detected galaxies are concentrated within the luminosity range of $\log \left(L_{K}\right)=6.0$ - 11.0. Such a segregation along $L_{K^{-}}$axis may be a source of specific bias in the statistics of apparent and intrinsic flattenings.

Figure 4 represents the distribution of 1049 LV galaxies with their apparent axial ratio and K-band luminosity. Galaxies detected and undetected in HI are indicated by filled and open small circles, respectively. The large circles and vertical rectangles correspond to the average value of $q$ and its dispersion in bins of $\Delta \log \left(L_{K}\right)=1.0$. The diagram shows a clear tendency for minimal values of $q$ to follow the parabolic envelope line with the absolute minimum of $q=0.05$ near $\log \left(L_{K}\right) \simeq 9$. Some dwarf gasless galaxies, like UMaI, deviate from the envelope line. They usually locate close to a massive neighboring galaxy (Martin et al. 2008), and their alongated shape can be caused by tidal perturbation.

Bradford et al. (2016) explored a sample of 930 isolated galaxies with axial ratios determined from the Sloan Digital Sky Survey (SDSS) and suggested for them a relation between true flattening and stellar mass of a galaxy as: $q_{0}=0.85-0.057 \times \log \left(M^{*} / M_{\odot}\right)$. According to this formula, the $q_{0}$ value grows monotonically from 0.2 for massive galaxies up to 0.5 for dwarfs. However, there is no place still for the common fact that the minimal values $q_{0} \simeq 0.05$ correspond just to the low mass galaxies of Sd-Sdm types.

Following Sanchez-Janssen et al. (2010), Lelli et al. (2016a) adopted the parabolic relation:

$$
q_{0}=5.2125-1.125 \log \left(M^{*}\right)+0.0625 \log \left(M^{*}\right)^{2},
$$

which reaches a minimum value of $q_{0}=0.15$ at $\log \left(M^{*}\right)=9.0$ and gives $q_{0}=0.4$ at $\log \left(M^{*}\right)$ equals 7.0 and 11.0. Both the relations by Bradford et al.(2016) and Lelli et al. (2016a) are shown in Figure 4 with dotted and dashed lines respectively.

Exploring properties of the LV galaxies and taking into account axial ratio statistics for ultraflat galaxies (Karachentseva et al. 2016), we re-parametrized the parabolic relation and found the optimum parameters:

$$
q_{0}=5.128-1.114 \log \left(M^{*}\right)+0.0612 \log \left(M^{*}\right)^{2} .
$$


This modified parabolic distribution, shown in Fig. 4 with solid line, reaches a minimum value of $q_{0}=0.059$ at $\log \left(M^{*}\right)=9.10$. We have to stress here that equations (4) and (5) should not be extrapolated for stellar masses below $\sim 10^{6} M_{\text {sun }}$ since they give unphysical results $\left(q_{0}>1\right)$.

If the distribution of galaxies over their intrinsic flatennings as a function of type or stellar mass is specified correctly, than, in the case of random spatial orientation of galaxy planes, the distribution of inclination angles will correspond to the $\sin i$-law (Yuan \& Zhu, 2004). Fig. 5 represents the distribution of Local Volume galaxies over inclination $i$ with step of $10^{\circ}$ under different assumptions (2)-(5) about intrinsic axial ratios. The solid line traces the expected distribution with uniformly random orientation of galaxy spin vectors over the sky. Vertical bars denote statistical errors.

As one can see from Fig. 5, the model (2) for galaxy distribution over intrinsic axial ratios as well as cases (4) and (5), reproduces the expected sin $i$-law much better than the RC2 scheme adopted in the CNG. The most significant deviations from the expected law in all the cases owe to underestimating the number of face-on galaxies: $i<20^{\circ}$. However, we have to exclude such galaxies from further consideration due to large uncertainties in corrected HI line width: $W_{50}^{c}=W_{50} / \sin i$. Another significant differences are seen in $i>70^{\circ}$ sector, but it has little effect on the corrected HI line width. One may suggest that the observed mismatch with the $\sin i$-law may be driven by $\mathrm{E}$, $\mathrm{S} 0$, and dSph galaxies. To test this hypothesis, we considered in the UNGC sample only galaxies with $M^{*}>10^{6} M_{\text {sun }}$ and $T>0$. As seen, this case shown by grey squares does not reduce the mismatch noticeably. Finally, the modified parabolic relation (5) connecting $q_{0}$ with $M^{*}$ is used by us in further study.

\section{Stellar Tully-Fisher relation for dwarfs.}

Considering different kinds of Tully-Fisher relation for the Local Volume, dominated by objects with low mass and luminosity, we have confined ourselves to the sample of galaxies with accurate distances (TRGB, Cep, SN, RR, CMD, HB). Then, we excluded from this sample two galaxies: Maffei2 and HIZSS03 with Galactic extinction of $A_{B}^{G}>3.0^{m}$ according to Schlafly \& Finkbeiner (2011). To reduce errors caused by the uncertainty in $W_{50}$ correction due to a galaxy inclination $i$, we further consider only galaxies with $i>i_{l i m}=45^{\circ}$. This condition decreases our sample from 296 to 206 . The analysis shows that stronger limitation does not change significantly the scatter in the TF diagram, but notably diminishes the sample volume.

Fig. 6 reproduces the classical Tully-Fisher diagram as the relation between the blue absolute magnitude of a galaxy, corrected for Galactic and internal extinction, and the HI line width for 206 galaxies taken from the LV database. The internal extinction was accounted according to Verheijen \& Sancisi (2001):

$$
A_{B}^{i}=\left[1.57+2.75\left(\log W_{50}-2.5\right)\right] \log (a / b),
$$


if $W_{50}>78 \mathrm{~km} \mathrm{~s}^{-1}$, otherwise $A_{B}^{i}=0$.

In the upper panel of Fig. 6, the measured width $W_{50}$ is plotted as abscissa covering the range from 10 to $500 \mathrm{~km} / \mathrm{s}$. The median value of $W_{50}$ for this sample amounts to $54 \mathrm{~km} / \mathrm{s}$ which is much lower than that for other TF-samples examined by Geha et al. 2006; Bradford et al. 2015, 2016; Brook et al. 2016; Sales et al. 2016; Papastergis et al. 2016. The $M_{B}$ vs $\log \left(W_{50}\right)$ relation is well described by the linear regression (a straight line) in the whole range of $W_{50}$ with a slope of -6.85 and a dispersion of $\sigma_{M}=1.06^{m}$. Here and below, the straight line in the TF diagram is a robust fit to the ensemble with errors in magnitudes. As seen, the dispersion increases appreciably while passing from massive spirals to dwarfs due mainly to observational errors. In the bottom panel, the corrected value of width, $W_{50}^{c}=W_{50} / \sin i$, is plotted on the horizontal axis. The view of the diagram has been changed subtly: the slope, -6.96 , and the scatter, $\sigma_{M}=1.10^{m}$, remained nearly the same.

To evaluate the behaviour of scatter on the TF diagram as a function of limiting inclination angle $i_{\text {lim }}$, we have consistently taken $i_{\text {lim }}=30,35, \ldots 80^{\circ}$. For each subsample we determined its new regression line and calculated dispersion $\sigma_{M}$ regarding to it. The results are presented in two panels of Fig. 7. The left panel corresponds to the case when the inclinations were determined from equation (2) via optical morphology, and the right panel illustrates the another manner with equations (5) accounting stellar masses. The numbers over the upper border of both panels indicate galaxy numbers in each subsample. The solid line and dashed line correspond to observed, $W_{50}$, and corrected, $W_{50}^{c}$, line width. These data show that giving away a half of the initial sample at $i_{\text {lim }}=(55-60)^{\circ}$, we may decrease the scatter only by $10-20 \%$. Also, the correction of line width for inclination does not improve noticeably a dispersion on the TF diagram inhabited mainly by dwarf galaxies. In other words, the $W_{50}$ correction for inclination in the realm of dwarfs looks as just a formal, optional procedure. This curious peculiar phenomenon was already noticed by Obreschkow \& Meyer (2013).

As noted by many authors (Aaronson et al. 1979; Aaronson et al. 1986; Giovanelli et al. 1997; Tully \& Pierce 2000), the dispersion in the TF diagram decreases systematically towards long wavelengths where the effects of internal extinction and star-bursts are not so significant. Near infrared $J, H, K_{s}$ photometry of galaxies fulfilled in the 2MASS all-sky survey (Jarett et al. 2000, 2003) provides background for constructing TF diagrams for spiral galaxies (Karachentsev et al. 2002). Yet, due to short expositions, the 2MASS survey has been found to be undersensitive for detecting low surface brightness objects, especially those ones having blue stellar population. About a half of the Local Volume galaxies stayed below the threshold of detectability. For many nearby dwarf galaxies, the integral $K$ magnitude obtained in 2MASS does not trace the appreciable contribution of the galaxy's periphery. According to the data on deep photometry of dwarf galaxies in $K$ and $H$ bands (McCall et al. 2012, Young et al. 2014), the typical error of these magnitudes for dwarfs in 2MASS is about $0.6^{m}$. If the accurate photometry was lacking, $K$ magnitudes in UNGC were estimated by us from $B$ magnitudes using the correlation between the mean color index $\langle B-K\rangle$ and morphological type of a galaxy (Jarrett et al. 2003): $\langle B-K\rangle=4.10$ for 
the early morphological types E, S0, Sa; $\langle B-K\rangle=2.35$ for the late types Sm, Im, BCD, Ir and $\langle B-K\rangle=4.60-0.25 \times T$ for intermediate types $T$ from 3 to 8 .

The $K$ band luminosity vs $W_{50}$ or $W_{50}^{c}$ relation for $206 \mathrm{LV}$ galaxies having accurately measured distances and $i>45^{\circ}$ is presented in the upper and lower panels of Fig. 8, respectively. The galaxies with $K_{s}$ magnitudes from 2MASS are labeled with filled circles, while galaxies with $K$ estimates made from the mean colour index $\langle B-K\rangle$ and morphological type $T$ are marked with open ones. These data show once again that galaxies without 2MASS photometry tend to reside in the left lower part of the diagram. The median value of $W_{50}$ for them is $36.5 \mathrm{~km} / \mathrm{s}$, while for galaxies with 2MASS magnitude their median is twice as much. Notably, both categories of objects - with 2MASS magnitudes and with $K$ estimates from the blue magnitude and morphological type follow the same regression line. Its slope, 2.87, and dispersion, 0.46 are actually the same in the both panels of Fig. 8 .

As it has been found by Noordermeer \& Verheijen (2007) and Schombert (2011) there is a systematic bias in 2MASS total magnitudes caused by some 2MASS surface photometry routines underestimating the luminosity of galaxies. This specific bias is especially significant for bright extended galaxies plentiful in the Local Volume. Since high-mass galaxies are star-dominated, a systematic underestimation of their K-luminosity may give shallower TF-slopes.

It should be also mentioned that the scheme of converting $B$ magnitudes to $K$ magnitudes adopted by Jarrett et al. (2003) is quite reliable in most cases. With a characteristic error of $\Delta T= \pm 1$ in morphological typing of a galaxy, the expected error in $K$ magnitude is $\pm 0.25^{m}$. However, for a special class of transition dwarf galaxies (dSph/dIr), a morphological typing mistake: $T=-1(\mathrm{dSph})$ else $T=10$ (dIr) leads to a leap in their $K$ magnitudes by $1.75^{m}$. Albeit, there are only five objects of this class in our sample: ESO 410-005, ESO 294-010, LGS-3, Antlia and HS 117.

\section{Gaseous TF-relation for dwarfs.}

Besides the stellar mass of a galaxy, its hydrogen mass,

$$
M_{H I}=2.356 \times 10^{5} \times D^{2} \times F(H I),
$$

also shows a tight correlation with HI line width. In the relation above the distance $D$ is expressed in Mpc and the flux $F(H I)$ in $\mathrm{Jy} \mathrm{km} \mathrm{s}^{-1}$. The distribution of 206 Local Volume galaxies over their hydrogen mass and HI line width $W_{50}$ is presented in the upper panel of Fig. 9. The regression line has a slope of 2.08, and the dispersion amounts to $\sigma\left(\log M_{H I}\right)=0.43$. Passing from $W_{50}$ to $W_{50}^{c}$ (middle panel) leaves unchanged the regression slope and the dispersion.

As known, some early-type galaxies and transition type dwarfs strongly deviate downward from the regression line in the gaseous TF diagram. On the bottom panel of Fig. 9 we excluded from our sample 16 gas-poor galaxies with colour index $m_{21}-K>6.0^{m}$, which approximately 
corresponds to the condition $M_{H I} / M^{*}<4 \%$. This procedure slightly increases the slope $(2.29)$ and decreases the dispersion (0.40). So we find that the TF dispersion of hydrogen mass for the late-type LV galaxies is of the same order as the TF dispersion of their stellar masses.

Fig. 9 outlines the upper limit for $M_{H I} /\left(W_{50}^{c}\right)^{2}$ value marked as dashed straight line in the lower panel. According to Zasov (1974), active star formation in disks of galaxies occurs usually near the threshold for their gravitational instability. This condition corresponds to the linear relation between the total mass of gas in a disk and its angular momentum: $M_{H I} \propto V_{m} \times A_{25}$, where $A_{25}$ is a standard linear diameter of disk. Taking into account the scaling relation $A_{25} \propto V_{m} \propto W_{50}$ for UCNG galaxies (Karachentsev et al. 2013), the presence of upper limit of $M_{H I} /\left(W_{50}^{c}\right)^{2}$ value agrees well with the idea of Zasov. Examples of the galaxies residing close to the dashed line are: NGC6744 $\left(W_{50}=323, \log \left(M_{H I}\right)=10.31, \log \left(L_{K}\right)=10.91\right)$ and $\operatorname{KDG} 177\left(W_{50}=30\right.$, $\left.\log \left(M_{H I}\right)=8.33, \log \left(L_{K}\right)=8.22\right)$.

\section{Baryonic TF-relation for the Local Volume galaxies.}

Over the last decade, a consensus has been achieved that a classical Tully-Fisher relation between the stellar mass (or luminosity) and the rotation amplitude, definable by dark halo mass, should also be contributed by the gas mass of a galaxy (McGaugh et al. 2000, Verheijen 2001, McGaugh 2005). Such a correction is particularly essential for galaxies of extremely low luminosities where the significant part of the gas has not yet been turned into stars. The TF relation for the total (stars plus gas) mass is known as baryonic Tully-Fisher relation (=bTF).

Many authors have explored the slope of bTF relation (Geha et al. 2006; Stark et al. 2009; Begum et al. 2008b; McGaugh et al. 2010; McGaugh 2012; Gurovich et al. 2010; Reyes et al. 2011; Torres-Flores et al. 2011; Catinella et al. 2012; Bradford et al. 2015; Lelli et al. 2016b; Papastergis et al. 2016). According to McGaugh \& Schombert (2015), bTF diagram $\log \left(M_{b a r}\right) \propto \beta \times \log \left(V_{m}\right)$ has a slope of $\beta=4.0$ in wide range of galaxy masses, which is not consistent with the expected value of $\beta \simeq 3.0$ for the standard cosmological model $\Lambda \mathrm{CDM}$. The steep $\beta \simeq 4$ slope has been also determined by Verheijen 2001, McGaugh 2005, Stark et al. 2009, Catinella et al. 2012, Lelli et al. 2016b, and Papastergis et al. 2016. Yet, other authors have obtained notably less steep $\beta$ values: 2.2 (Begum et al. 2008b), 2.1 (McCall et al. 2012), 2.5 (Kirby et al. 2012). As it was discussed by Brook et al. (2016), Brook \& Shankar (2016) and Bradford et al. (2016), the slope value $\beta$ can vary from 2 up to 4 depending on methodological details of converting observational values $W_{50}, W_{20}$ or $V_{\text {flat }}$ to the amplitude of rotation curve, $V_{\text {rot }}$. Also, as the most gas-rich dwarf galaxies follow the shallow relation $M_{H I} \propto W_{50}^{2.2}$, than the high or low proportion of irregular dwarfs in the sample can appreciably affect the slope $\beta$. Anyway, it must be remembered that the $W_{50}$ line widths derived from spatially unresolved (single dish) observations give systematically shallower TF relation slope than flat rotational velocities, $V_{\text {flat }}$, from interferometric HI observations. The reason for this is due to the known effect that dwarf galaxies have slowly rising rotation curves and reach $V_{\text {flat }}$ only in the outermost regions, where the HI surface densities are low and the $\mathrm{S} / \mathrm{N}$ ratio 
goes down (Swaters et al. 2009; Lelli et al. 2014).

To determine the baryonic mass of a galaxy

$$
M_{\text {bar }}=M^{*}+M_{\text {gas }}=\Upsilon_{K}^{*} \times L_{K}+\eta \times M_{H I},
$$

two parameters should be held fixed: stellar-mass-to- $K$-luminosity ratio (or a similar ratio for any other photometric band) and the $\eta$ factor used for converting neutral hydrogen mass to the total gas mass. The published values of $\Upsilon^{*}=M^{*} / L_{K}$ stay within the range of $[0.5-1.0] M_{\odot} / L_{\odot}$. They are based on different models of synthetic stellar populations. According to analysis fulfilled by McGaugh \& Schombert (2014), the optimal value of $\Upsilon_{K}^{*}$ is close to $0.6 M_{\odot} / L_{\odot}$, though Just et al. (2015) present the value of $M^{*} / L_{K}=(0.31 \pm 0.02) M_{\odot} / L_{\odot}$ for the solar vicinity from Hipparcos data.

The factor $\eta$ is usually set to 1.33 taking into account the correction for Helium abundance. According to Fukugita \& Peebles (2004), adding molecular gas to consideration leads to a value of $\eta=1.85$. However, this higher factor is typical only for disks of massive galaxies. The data by Young \& Knezek (1989) and McGaugh \& de Blok (1997) show that molecular-to-atomic hydrogen mass ratio decreases rapidly towards dwarf galaxies as

$$
M_{H 2} / M_{H I}=3.7-0.8 \times T+0.043 \times T^{2},
$$

and for dwarfs $(T \geq 8)$ it does not exceed 0.05 .

Yet, the situation with $\eta$ value remains rather uncertain. It is commonly known (see, for example, Fukugita \& Peebles 2004) that the observable quantity of baryons in galaxies and in hot intergalactic gas of clusters $(\sim 0.005 \Omega)$ is by an order of magnitude less than expected from the standard cosmological model of nucleosynthesis $(0.045 \Omega)$. It is suggested that the bulk of baryons escaping from observations is distributed as warm or hot intergalactic gas, which can be partially associated with galaxies. Attempts of detecting hot gaseous coronas were undertaken based on observations of absorbtion lines OVII and OVIII in quasar spectra (Stocke et al. 2013). According to Miller \& Bregman (2015), the mass of ionized gas around the Milky Way is $(3.8 \pm 0.3) \times 10^{9} M_{\odot}$ within the radius of $50 \mathrm{kpc}$, and $(4.3 \pm 0.9) \times 10^{10} M_{\odot}$ within $250 \mathrm{kpc}$. The last value is matching with the total stellar mass of the Milky Way $\left(6 \times 10^{10} M_{\odot}\right)$ and is several times more than the mass of neutral hydrogen in our Galaxy. If such warm gaseous halo is typical for other massive and dwarf galaxies, than the dimensionless factor $\eta$ can amount to $\sim 5$. Varying $\eta$ parameter, Pfenniger \& Revaz (2005) and Begum et al. (2008b) concluded that the minimum dispersion in bTF diagram is reached within a wide range of $\eta=(3-10)$. This result implicitly confirms that a large number of hot baryons may be associated with galaxies beyond the known amount of neutral gas in them.

The distributions of the Local Volume galaxies over baryonic mass and HI line width, $W_{50}$ or $W_{50}^{c}$, are presented in the upper and lower panels of Fig. 10. For factors converting $M^{*}$ and $M_{H I}$ to baryonic mass we have adopted $\Upsilon_{K}^{*}=0.60$ (McGaugh \& Schombert 2014) and $\eta=1.33$. The slope of the regression line and the dispersion are $\beta=2.55, \sigma=0.36$ (for $W_{50}$ ) and $\beta=2.61, \sigma=0.38$ (for $W_{50}^{c}$ ), respectively. 
As we have already mentioned, the correction for inclination in the case of dwarf galaxies does not play any significant role, so we included into our analysis another 56 faint dwarf galaxies with $M_{B}>-16.0^{m}$ and inclinations $i<45^{\circ}$ (marked as crosses in Fig. 11). We added also 69 mainly dwarf galaxies with distance estimates (mem) obtained from their membership in the known groups. The typical distance error for them is $\sim 17 \%$ or 0.14 dex in the scale of $M_{b a r}$, which is three times less than the observed TF scatter. These objects with arbitrary inclinations if $M_{B}>-16.0^{m}$, or $i>45^{\circ}$ otherwise, are labeled as open diamonds. The regression line for the complemented sample of 331 galaxies has a slope of $\beta=2.49$ and dispersion of $\sigma\left(\log M_{b a r}\right)=0.38$.

To optimize the choice of $\Upsilon_{K}^{*}$ and $\eta$ parameters for the Local Volume galaxies we calculated their baryonic mass with various values of $\Upsilon_{K}^{*}$ from 0.40 to 1.00 and $\eta$ from 1.33 to 5.0. The RMS deviations from the regression line for each combination of parameters are presented as matrix in Table 2. The differences in $\sigma\left(\Upsilon_{K}^{*}, \eta\right)$ turn out to be small. The minimum value of dispersion, 0.352 , is found along the matrix diagonal going from $\Upsilon_{K}^{*}=0.40$ and $\eta=2.50$ to $\Upsilon_{K}^{*}=1.00$ and $\eta=5.0$. This result could be interpreted in favour of the presence of dark (warm) gas around the LV galaxies, however, the TF-scatter in the current sample is rather dominated by various observational errors. Hence, its variations can not be really used to constrain intrinsic galaxy properties like $\Upsilon_{K}^{*}$ and $\eta$.

The obtained parameters of regression lines for the different kinds of TF relations are summarized in Table 3.

\section{A secondary parameter in the bTF-diagram.}

In literature, one can find different attempts of reducing the scatter of galaxies in the TullyFisher diagram by introducing some additional parameters (Kashibadze 2008; Kudrya et al. 2009; McCall et al. 2012). Apparently, these parameters should be chosen as been independent on galaxy distance. The examples of such variables are: surface brightness of a galaxy, its gas fraction index, morphological type, apparent axial ratio, specific star formation rate, and isolation index. Strictly speaking, all of them still show a slight dependence on distance due to different effects of observational selection. It should be also kept in mind that the listed parameters may be mutually

Table 2: Observational scatter in the baryonic Tully-Fisher diagram as a function of adopted values $\Upsilon^{*}$ and $\eta$.

\begin{tabular}{ccccccc}
\hline$\eta$ & 1.33 & 1.85 & 2.50 & 3.00 & 4.00 & 5.00 \\
$\Upsilon^{*}$ & & & & & & \\
\hline 0.40 & 0.357 & 0.353 & 0.352 & 0.353 & 0.356 & 0.360 \\
0.50 & 0.362 & 0.355 & 0.352 & 0.352 & 0.353 & 0.356 \\
0.60 & 0.366 & 0.358 & 0.354 & 0.352 & 0.352 & 0.354 \\
0.75 & 0.371 & 0.363 & 0.357 & 0.354 & 0.352 & 0.352 \\
1.00 & 0.380 & 0.370 & 0.363 & 0.359 & 0.354 & 0.352 \\
\hline
\end{tabular}


correlated. Nevertheless, the search for a secondary parameter in the bTF relation offers a chance to improve the accuracy of measuring galaxy distances by such approach (if the application of the corresponding parameter does not drop the sample number essentially).

As candidates, we have tested the following parameters available in the UNGC.

1) The mean surface brightness in $B$ band within the Holmberg isophote,

$$
S B=B_{T}^{c}+5 \log a_{26}+8.63
$$

where the total apparent magnitude $B_{T}^{c}$ and the angular diameter in arcminutes, $a_{26}$, are corrected for extinction and inclination effects.

2) The gas fraction index $\left(B_{T}^{c}-m_{21}\right)$.

3) The specific star formation rate,

$$
P=\log (S F R)-\log L_{K}+10.14
$$

normalized to the age of Universe. Here, the integral star formation rate was defined either from apparent magnitude in far ultraviolet based on GALEX data (Gil de Paz et al. 2007)

$$
\log \left(S F R\left[M_{\odot} \times y r^{-1}\right]\right)=2.78-0.4 m_{F U V}^{c}+2 \log D
$$

or via $H \alpha$ flux (Karachentsev \& Kaisina 2014)

$$
\log \left(S F R\left[M_{\odot} \times y r^{-1}\right]\right)=8.98+\log F_{c}(H \alpha)+2 \log D
$$

with considering galactic and internal extinction: $A(F U V)=1.93\left(A_{B}^{G}+A_{B}^{i}\right)$ and $A\left(H_{\alpha}\right)=$ $0.538\left(A_{B}^{G}+A_{B}^{i}\right)$, where $A_{B}^{G}$ from Schlafly \& Finkbeiner (2011) and $A_{B}^{i}$ from equation (6).

All three parameters are independent from the distance $D$, and their values are available in our database for most LV galaxies. We also tested two parameters, characterizing the local environment of a galaxy: the index of isolation

$$
\Theta_{1}=\max \left[\log \left(M_{n} / D_{n}^{3}\right)\right]+C, \quad n=1,2, \ldots N,
$$

which distinguishes among the plenty of nearby galaxies the most significant neighbor (the main disturber $=\mathrm{MD}$ ), whose tidal force, $F_{n} \sim M_{n} / D_{n}^{3}$ dominates all other neighbors (Karachentsev et al, 2013). Here, $D_{n}$ is the $3 \mathrm{D}$ separation of a neighboring galaxy, and the value of constant $C=-10.96$ was chosen so that at $\Theta_{1}=0$ the neighbor $\mathrm{n}$ is located on the zero velocity sphere relative to the MD. At that, the galaxies with $\Theta_{1}>0$ turned out to be members of a certain group, and the negative values of $\Theta_{1}$ corresponded to isolated galaxies. The tidal index $\Theta_{1}$ or actually a stellar density contrast, contributed by one, most important neighbor, i.e. MD, can significantly change with time due to orbital motions of galaxies. That is why we also used another isolation index 


$$
\Theta_{5}=\log \left(\sum_{n=1}^{5} M_{n} / D_{n}^{3}\right)+C,
$$

which is the sum of the density contrasts produced by five most important neighbors. The value of constant $\mathrm{C}$ here is the same as in Equation (7).

Four panels of Fig. 12 show the relation between a galaxy deviation from the regression line and the value of each of four mentioned parameters. The galaxy subsample designations are the same as in Fig. 11. Table 4 provides the values of the regression slope $\beta$ with its error, the RMS scatter $\sigma$, and the Pearson $(\rho)$ and Kendall $(\tau)$ correlation coefficients with their statistical significance p-values. No correlation is statistically significant since absolute values of all correlation coefficients are well below 0.2 . The observed lack of environmental dependence of the bTF relation looks to be a rather unexpected result.

\section{Bald (dSph) dwarfs in the TF-diagram.}

As it is seen from Figures 3 \& 4, only 656 out of 1049 Local Volume objects have been detected in HI. The fraction of objects without HI-data is growing rapidly towards the low mass galaxies. About a third of the HI-undetected objects have been never observed in HI because they are located outside the HIPASS and ALFALFA surveys. The majority of the remainig undetected objects are dwarf spheroidal (dSph) and dwarf elliptical (dE) galaxies with a small admixture of transition dwarfs (dTr) and outlying globular clusters around M31. The relative number of true gas-poor $\mathrm{dSph}$ and $\mathrm{dE}$ galaxies (bald dwarfs) in the LV sample amounts to $\sim 20 \%$. However, this quantity is rather uncertain. No evidence of gas or structure details, as well as low surface brightness, make dSphs very difficult to determine their distances and radial velocities. Actually, most their distances were estimated from supposed membership in the known groups. There is no any deep systematic survey for dSphs over the whole sky. In practice, dSph galaxies can be identified as the Local Volume objects only after resolving them into stars, which is possible to reach with the existing ground-based telescopes just within the Local Group and its immediate surroundings.

At present, radial velocities and stellar radial velocity dispersion, $\sigma_{*}$, are measured for $69 \mathrm{dSph}$ and $2 \mathrm{dE}$ satellites of the Milky Way and M31. We compiled a summary of their $\sigma_{*}$ and stellar masses, based on the data from Collins et al. (2013, 2014) and Wheeler et al. (2015) with additions from Walker et al.(2016) and Irwin \& Tolstoy (2002). Their distribution is presented in Fig. 13, where horizontal bars indicate the measurement errors. As one can see, for many tiny dwarfs velocity dispersion is comparable with its typical error of $\sim(2-5) \mathrm{km} / \mathrm{s}$.

Then, we incorporated the data on dSph and dE companions of the Milky Way and M31 into the bTF diagram, adopting the value $W_{*}=2 \sqrt{2 \ln 2} \times \sigma_{*}$ as an analogue of the line width $W_{50}$ for the Gaussian function, and setting $M_{b a r}=M^{*}$. The combined bTF diagram for 404 late-type and 
Table 3: The measured values of slope $(\beta)$, zero-point $(\mathrm{C})$ and dispersion $(\sigma)$ for the various types of Tully-Fisher relations.

\begin{tabular}{ccccc}
\hline LV sample & $\mathrm{N}$ & $\beta$ & $\mathrm{C}$ & $\sigma$ \\
\hline$M_{B}$ vs $W_{50}$, TRGB,$i>45$ & 206 & -6.85 & -2.78 & 1.06 \\
$M_{B}$ vs $W_{50}^{c}$, TRGB,$i>45$ & 206 & -6.96 & -2.19 & 1.10 \\
$L_{K}$ vs $W_{50}$, TRGB, $i>45$ & 206 & 2.87 & 3.16 & 0.46 \\
$L_{K}$ vs $W_{50}^{c}$, TRGB, $i>45$ & 206 & 2.91 & 2.91 & 0.47 \\
$M_{H I}$ vs $W_{50}$, TRGB, $i>45$ & 206 & 2.08 & 4.24 & 0.43 \\
$M_{H I}$ vs $W_{50}^{c}$, TRGB, $i>45$ & 206 & 2.14 & 4.01 & 0.44 \\
$M_{H I}$ vs $W_{50}^{c}, M_{H I} / M^{*}>0.04$ & 190 & 2.28 & 3.79 & 0.40 \\
$M_{b a r}$ vs $W_{50}$ & 206 & 2.55 & 3.91 & 0.36 \\
$M_{b a r}$ vs $W_{50}^{c}$ & 206 & 2.61 & 3.64 & 0.38 \\
$M_{b a r}$ vs $W_{50}$, extended & 331 & 2.49 & 3.97 & 0.38 \\
\hline
\end{tabular}

Table 4: Various residual non-correlations

\begin{tabular}{lrrrrrrr}
\hline \multicolumn{1}{c}{$\beta$} & \pm & $\sigma$ & $\rho$ & $p_{\rho}$ & $\tau$ & $p_{\tau}$ \\
\hline $\mathrm{SB}$ & -0.078 & 0.023 & 0.376 & -0.160 & 0.003 & -0.107 & 0.004 \\
$B_{T}^{c}-m_{21}$ & -0.034 & 0.015 & 0.380 & -0.077 & 0.164 & -0.082 & 0.027 \\
$\mathrm{P}$ & 0.006 & 0.040 & 0.380 & 0.008 & 0.891 & -0.008 & 0.825 \\
$\Theta_{1}$ & 0.008 & 0.014 & 0.380 & 0.019 & 0.732 & 0.038 & 0.301 \\
$\Theta_{5}$ & 0.008 & 0.014 & 0.380 & 0.019 & 0.742 & 0.038 & 0.301 \\
$\log (b / a)$ & 0.044 & 0.102 & 0.383 & 0.018 & 0.746 & -0.029 & 0.430 \\
\hline
\end{tabular}


early-type galaxies is shown in Fig. 14. The gas-poor dwarfs are indicated by red stars. As seen, the population of dSphs forms a wide tail curved downward with much steeper bTF slope. An essential part of their scatter is caused by errors in $\sigma_{*}$ themselves. In the range of $W>20 \mathrm{~km} / \mathrm{s}$, most the early-type dwarfs follow nearly the same bTF relation as gas-rich galaxies, that has been found by McGaugh \& Wolf (2010) and den Heijer et al. (2015). Although in our opinion, the dSphs show at average lower masses than dIrs. Being improved in $\sigma_{*}$ errors, this diagram will be useful to test different scenarios describing transformation of late-type dwarfs into quenched dSphs. McGaugh \& Wolf (2010) also noted that the deviations of dSphs from bTF relation are considerably more susceptible to tidal effects in Modified Newtonian dynamics (MOND) than in the standard dark matter paradigm.

The raw observational data on 402 galaxies of the Local Volume, including $71 \mathrm{dSphs,}$ are compiled in Table 5. Its columns contain: (1) galaxy name; (2) equatorial coordinates; (3) morphological type; (4) apparent axes ratio; (5) HI line width $W_{50}$ (or $W^{*}$, marked by asterisk); (6) galaxy distance in Mpc and the method used to determine it; (7) logarithm of the $K$-band luminosity in solar units; (8) logarithm of hydrogen mass in solar masses; (9) logarithm of baryonic mass with parameters: $\Upsilon^{*}=0.60, \eta=1.33$. The full text of the Table is available online in http://www.sao.ru/lv/lvgdb/.

\section{Discussion and concluding remarks.}

Estimating the budget of errors responsible for the observed scatter of galaxies in the bTF diagram we have taken into account five different sources of the scatter.

1) Many dwarf galaxies in the UNGC sample have eye-ball estimates of apparent $B$ magnitude with an accuracy of $\sim 0.5^{m}$. An additional error appears while converting $B$ magnitudes to $K$ ones. Suggesting the total error $\sigma(K) \simeq 0.6^{m}$, we obtain its contribution to the baryonic mass error as $\sigma\left(\log M_{\text {bar }}\right)=0.25$.

2) Inspection of the HI line width errors in our sample yields the average mean-square value of $17 \%$ or 0.07 dex. At the slope of the regression line as $\beta=2.6$, the contribution of $\sigma\left(W_{50}\right)$ errors accounts to $\sim 0.18$ dex, i.e. slightly less than the contribution of photometric errors.

3) According to Meidt et al. (2014) the scatter on the stellar mass-to-NIR luminosity ratio for galaxies amounts $\sim 0.11$ dex.

4) The uncertainty in distances for the LV objects $(\sim 10 \%)$ produces the error in calculating baryonic mass of $\sim 0.08$ dex, being lower than from previous observables.

5) The typical observed error of HI flux for our sample galaxies is $\sim 20 \%$, that gives $\sim 0.08$ dex on the bTF plot.

Therefore, after adding quadratic errors, the resulting expected error from these observables 
is $\sigma\left(\log M_{b a r}\right)=0.35$. Performing its quadratic subtraction from the observed scatter of galaxies in the bTF diagram (0.38 dex), we obtain the intrinsic (cosmic) scatter in the baryonic mass to be $\sigma\left(\log M_{\text {bar }}\right)_{\text {cosmic }}=0.15$. A part of this residual scatter is caused by imperfect recipe used to account inclinations of very low mass galaxies often having irregular shapes.

Our evaluation of the observed ( 0.38 dex) and intrinsic ( 0.15 dex) scatter in $M_{b a r}$ for the sample of 331 nearby dwarf galaxies can be compared with other estimates from literature. It should be kept in mind, that in most papers devoted to baryonic Tully-Fisher relation the analysis is based on samples consisting of spiral galaxies with typical HI line width $W_{50}>100 \mathrm{~km} / \mathrm{s}$, where dwarf galaxies are only a kind of supplement.

McGaugh (2012) selected 47 gas-rich galaxies in the range of $\log \left(M_{b a r}\right)=[7-11] \log \left(M_{\odot}\right)$ and found for them the observed scatter of 0.24 dex. Just the same observed scatter, 0.24 dex, has been obtained by McCall et al. (2012) based on a deep $K$-band photometry of 19 late-type dwarf galaxies of the Local Volume. Their sample has a median value of $\log \left(M_{b a r}\right)$ about $8.3 \log \left(M_{\odot}\right)$. Lelli et al. (2016b) studied a sample of 118 spiral and irregular galaxies with a high quality data on their photometry and extended HI rotation curves. For this sample covering the baryonic mass range of $\log \left(M_{b a r}\right)=[8.0-11.5] \log \left(M_{\odot}\right)$ the authors found the observed scatter of 0.22 dex and estimated the intrinsic scatter as 0.10 dex. Almost the same quantities of the observed and intrinsic scatter were obtained by McGaugh \& Schombert (2015) for a sample of $26 \mathrm{~S}$ and dIr galaxies with the median $V_{\text {flat }}$ velocity of $130 \mathrm{~km} / \mathrm{s}$. The sample of 97 gas-rich spiral and irregular galaxies selected by Papastergis et al. (2016) probes the bTF relation over the $\log \left(M_{b a r}\right)=[8.5-10.5] \log \left(M_{\odot}\right)$. The authors derived for them the observed scatter of baryonic mass to be $\sim 0.22$ dex. A close value, 0.25 dex, was found by Bradford et al. (2015) for a sample of 148 gas-rich galaxies from SDSS with stellar masses ranging between $10^{7}$ and $10^{9.5} M_{\odot}$. Recently, Bradford et al. (2016) performed a comprehensive analysis of slope and dispersion of the bTF relation using a sample of 930 isolated galaxies that have accurate photometry from the Sloan Digital Sky Survey (SDSS). Their sample extends over the baryonic mass $\log \left(M_{b a r}\right)=[7.4-11.3] \log \left(M_{\odot}\right)$. The observed bTF scatter for the total sample is found to be 0.25 dex. In the same time, the sub-sample of 271 low-mass galaxies with $W_{20}<200 \mathrm{~km} / \mathrm{s}$ is characterized by larger scatter of 0.41 dex.

We suppose that improving the measurement accuracy of $K$ magnitudes as well as $W_{50}$ widths, expected in forthcoming deep optical, NIR, and HI sky surveys, may reduce the observed scatter of $\log \left(M_{b a r}\right)$ for the low-mass dwarfs till 0.20 dex, and thereby achieve the accuracy of bTF distances for them $\sim 0.10$ dex, i.e. $\sim 25 \%$. This improvement will be important to refine the peculiar velocity field in the Local Universe.

Acknowledgements.

We thank the anonymous referee fo numerous helpful comments that essentially improved the paper. We thank Jayaram Chengalur, Brent Tully, Valentina Karachentseva and Dmitry Makarov for fruitful discussions. This work was supported by the Russian Science Foundation, grant 1412-00965. Support for proposals GO 12546, 14636 was provided by NASA through grants from 
the Space Telescope Science Institute, which is operated by the Association of Universities for Research in Astronomy, Inc., under NASA contract NAS526555. Observations with the Russian 6-meter telescope BTA and Indian Giant Meterwave Radio Telescope GMRT have been supported by the Russian Foundation for Basic Research grant 15-52-45004 (IND-RUS).

R e f e r e n c e s

Aaronson M., Huchra J., Mould J. 1979, ApJ, 229, 1

Aaronson M., Bothun G., Mould J. et al. 1986, ApJ, 302, 536

Balkowski C., Bottinelli L., Chamaraux P., et al, 1974, $A$ \& $A, 34,43$

Begum A., Chengalur J.N., Karachentsev I.D., Sharina M.E., Kaisin S.S. 2008a, MNRAS, 386, 1667

Begum A., Chengalur J.N., Karachentsev I.D., Sharina M.E. 2008b, MNRAS, 386, 138

Bell E.F., McIntosh D.H., Katz N., Weinberg M.D. 2003, ApJS, 149, 289

Bradford J.D., Geha M.C., Blanton M.R. 2015, ApJ, 809, 146

Bradford J.D., Geha M.C., van den Bosch F.C., 2016, 1602.02757

Brook C.B., Santos-Santos I., Stinson G., 2016, MNRAS, 459, 638

Brook C.B., Shankar F., 2016, MNRAS, 455, 3841

Catinella B., Kauffmann G., Schiminovich D., et al. 2012, MNRAS, 420, 1959

Collins M.L., Chapman S.C., Rich R.M., et al. 2013, ApJ, 768, 172

Collins M.L., Chapman S.C., Rich R.M., et al. 2014, ApJ, 783, 7

de Vaucouleurs, G., de Vaucouleurs, A., \& Corwin, H. 1976, Second Reference Catalogue of Bright Galaxies, Texas, Austin

den Heijer M., Oosterloo T.A., Serra P., et al. 2015, A\&A, 581A, 98

Fukugita M., Peebles P.J.E. 2004, ApJ, 616, 643

Garcia-Ruiz \& Sancisi 2002, A $\& A, 394,769$

Geha M., Blanton M.R., Masjedi M., West A.A. 2006, ApJ, 653, 240

Gil de Paz, A., Boissier, S., Madore, B. F., et al. 2007, ApJS, 173, 185

Giovanelli R., Haynes M.P., Herter T., et al. 1997, AJ, 113, 53

Giovanelli R., Haynes M.P., Kent B.R. et al. 2005, AJ, 130, 2598

Gurovich S., Freeman K., Jerjen H., et al. 2010, AJ, 140, 663 
Haynes M.P., Giovanelli R., Martin A.M. et al. 2011,AJ, 142, 170

Huchtmeier, W.K., Karachentsev, I.D., Karachentseva, V.E. \& Ehle M. 2000, A\&GAS, 141, 469

Huchtmeier W.K., Karachentsev I.D., Karachentseva V.E. 2001, A\&\&A, 377, 801

Huchtmeier W.K., Karachentsev I.D., Karachentseva V.E. 2003, A\&\&A, 401, 483

Irwin M., Tolstoy E. 2002, MNRAS, 336, 643

Jarrett, T.N., Chester, T., Cutri R. et al. 2000, AJ, 119, 2498

Jarrett T.H., Chester T., Cutri R., Schneider S. E., Huchra J. P. 2003, AJ, 125, 525

Just A., Fuchs B., Jahreiss H., et al. 2015, MNRAS, 451, 149

Kaisina E.I., Makarov D.I., Karachentsev, I.D., Kaisin S.S. 2012, AstBu, 67, 115

Karachentsev I.D., 1989, AJ, 97, 1566

Karachentsev I.D., Karachentseva V.E., Kudrya Yu.N., 1999, Astronomy Letters, 25, 1

Karachentsev I.D., Mitronova S.N., Karachentseva V.E., Kudrya Yu.N., Jarrett T.H. 2002, $A \mathscr{E} A, 396,431$

Karachentsev, I.D., Karachentseva, V.E., Huchtmeier, W.K., Makarov, D.I. 2004, AJ, 127, 2031 (CNG)

Karachentsev I.D., Makarov D.I., Kaisina E.I. 2013, AJ, 145, 101 (UNGC)

Karachentsev, I.D., Kaisina, E.I. 2014, AJ, 146, 46

Karachentseva V.E., Kudrya Y.N., Karachentsev I.D., et al. 2016, AstBull, 71, 1

Kashibadze O.G., 2008, Ap, 51, 336

Kirby E.M., Koribalski B., Jerjen H., Sanchez A.L. 2012, MNRAS, 420, 2924

Koribalski B.S., Staveley-Smith L., Kilborn V.A. et al. 2004, AJ, 128, 16

Kovaĉ K., Oosterloo T.A., van der Hulst J.M. 2009, MNRAS, 400, 743

Kraan-Korteweg, R.C. \& Tammann, G.A. 1979, AN, 300, 181

Kudrya Yu. N. Karachentseva V.E., Karachentsev I.D., et al. 2009, Ap, 52, 367

Lelli F., Verheijen M., Fraternali F., 2014, A \& A, 566A, 71

Lelli F., McGaugh S.S., Schombert J.M., Pawlowski M.S., 2016a, Ap, 827L, 19

Lelli F., McGaugh S.S., Schombert J.M. 2016b, ApJ, 816, L14

Martin N.F., de Jong J.T.A., Rix H.W., 2008, ApJ, 684, 1075 
McCall M.L., Vaduvescu O., Nunez F.P., et al. 2012, A\&A, 540A, 49

McGaugh S.S. 2005, ApJ, 632, 859

McGaugh S.S. 2012, AJ, 143, 40

McGaugh S.S., Schombert J.M., Bothun G.D., de Blok W.J. 2000, ApJ, 533, L99

McGaugh S.S., Schombert J.M., de Blok W.J., Zagursky J. 2010, ApJ, 708L, 14

McGaugh, S.S., \& de Blok, W.J.G. 1997, ApJ, 481, 689

McGaugh S.S., Schombert J.M. 2014, AJ, 148, 77

McGaugh S.S., Schombert J.M. 2015, ApJ, 802, 18

McGaugh S.S., Wolf J., 2010, ApJ, 722, 248

Meidt S.E., Schinnerer E., van de Ven G., et al. 2014, ApJ, 788, 144

Meyer M.J., Zwaan M.A., Webster R.L. et al. 2004, MNRAS, 350, 1195

Miller M.J., Bregman J.N. 2015, ApJ, 800, 14

Noordermeer E., Verheijen M.A.W., 2007, MNRAS, 381, 1463

Obreschkow D., Meyer M. 2013, ApJ, 777, 140

Papastergis E., Adams E.A.K., van der Hulst J.M. 2016, a-ph/1602.09087

Paturel G., Andernach H., Bottinelli L., et al. 1997, A\&AS, 124, 109

Pfenniger D., Revaz Y. 2005, A\&3A, 431, 511

Reyes R., Mandelbaum R., Gunn J.E., et al. 2011, MNRAS, 417, 2347

Roberts M.S. 1969, AJ, 74, 859

Roychowdhury S., Chengalur J.N., Begum A., Karachentsev I.D. 2010, MNRAS, 404, L60

Roychowdhury S., Chengalur J.N., Karachentsev I.D., Kaisina E.I. 2013, MNRAS, 436L, 104

Sales V.L., Navarro J.F., Oman K. et al., 2016, a-ph/1602.02155

Sanchez-Janssen R., Mendez-Abreu J., Aguerri J.A.L., 2010, MNRAS, 406L, 65

Sanchez-Janssen R., Ferrarese L., MacArthur L.A., et al, 2016, ApJ, 820, 69

Schlafly, E. F., \& Finkbeiner, D. P. 2011, ApJ, 737, 103

Schombert J., 2011, a-ph/1107.1728

Stark D.V., McGaugh S.S., Swaters R.A. 2009, AJ, 138, 392

Staveley-Smith L., Kraan-Korteweg R.C., Schroder A.C., et al. 2016, AJ, 151, 52 
Stocke J.T., Keeney B.A., Danforth C.W., et al. 2013, ApJ, 763, 148

Swaters R.A., Sancisi R., van Albada T.S., van der Hulst J.M., 2009, A\& A, 493, 871

Tonry, J.L., Dressler, A., Blakeslee, J.P. et al. 2001, ApJ, 546, 681

Torres-Flores S., Epinat B., Amram P. et al. 2011, MNRAS, 416, 1936

Tully R.B., Fisher J.R. 1977, A\&A, 54, 661

Tully R.B., Pierce M.J. 2000, ApJ, 533, 744

Verheijen M.A.W. 2001, ApJ, 563, 694

Verheijen M.A.W., Sancisi R., 2001, A \& A, 370, 765

Walker M.G., Mateo M., Olszewski E.W., et al. 2016, ApJ, 819, 53

Wheeler C., Pace A.B., Bullock J.S. et al. 2015, a-ph/1511.01095

Wong O.I., Ryan-Weber E.V., Garcia-Appadoo et al. 2006, MNRAS, 371, 1855

Young T., Jerjen H., Lopez-Sanchez A.R., Koribalski B.S. 2014, MNRAS, 444, 3052

Young J.S., Knezek P.M. 1989, ApJ, 347L, 55

Yuan Q.R., Zhu C.X., 2004, Chinese Astr. Astrophys., 28, 127

Yun M.S., Ho P.T.P., Lo K.Y., 1994, Nature, 372, 6506, 530

Zasov A.V. 1974, AZh, 51, 1225 


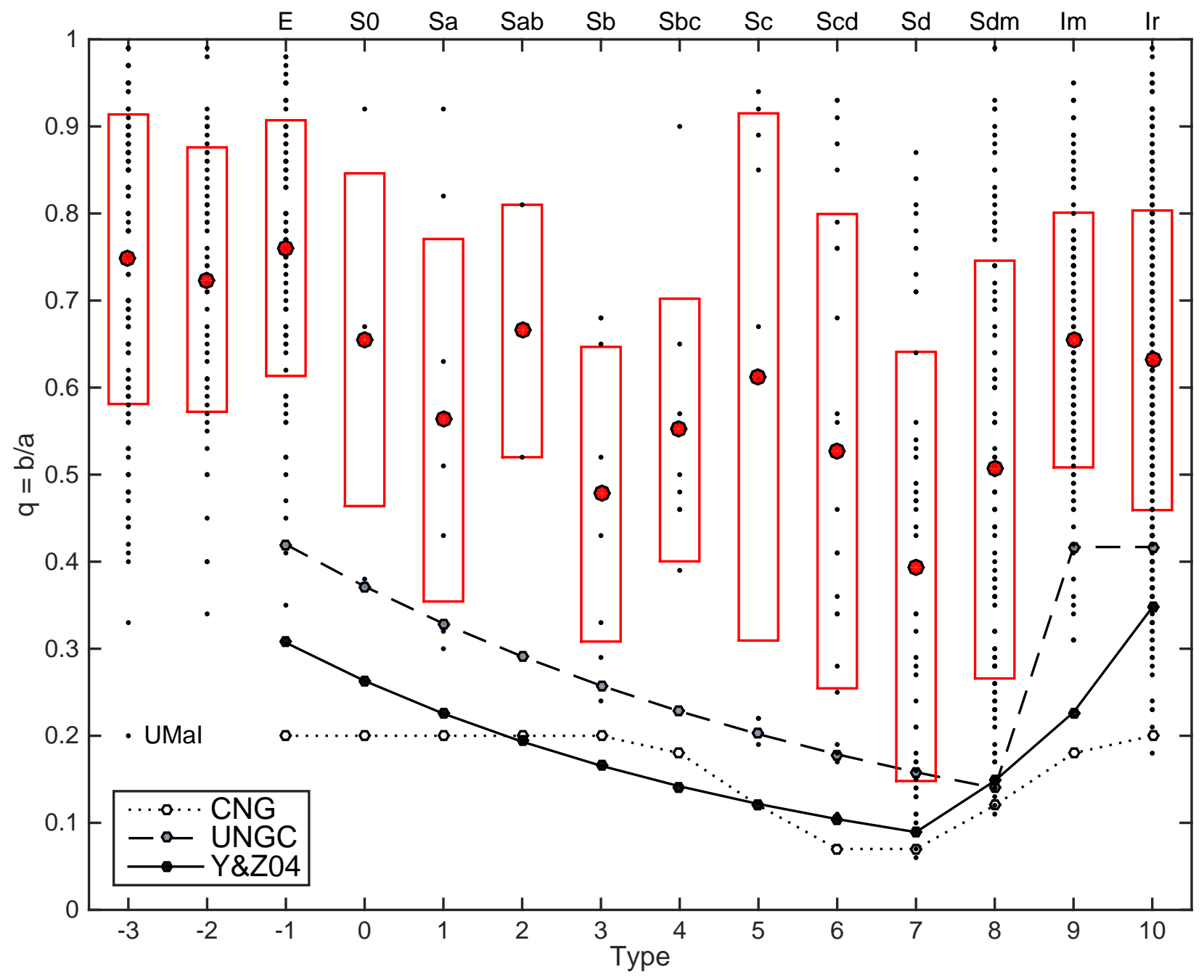

Fig. 1. - Apparent axial ratio of the Local Volume galaxies vs. their morphological type in de Vaucouleurs scale. The large circles and vertical rectangles indicate the mean values and the standard deviations for each type. The dotted, dashed and solid lines present intrinsic axial ratios for different morphological types as adopted in the Catalog of Neighboring Galaxies, the Updated Nearby Galaxy Catalog and as assumed by Yuan \& Zhu (2004), respectively. 


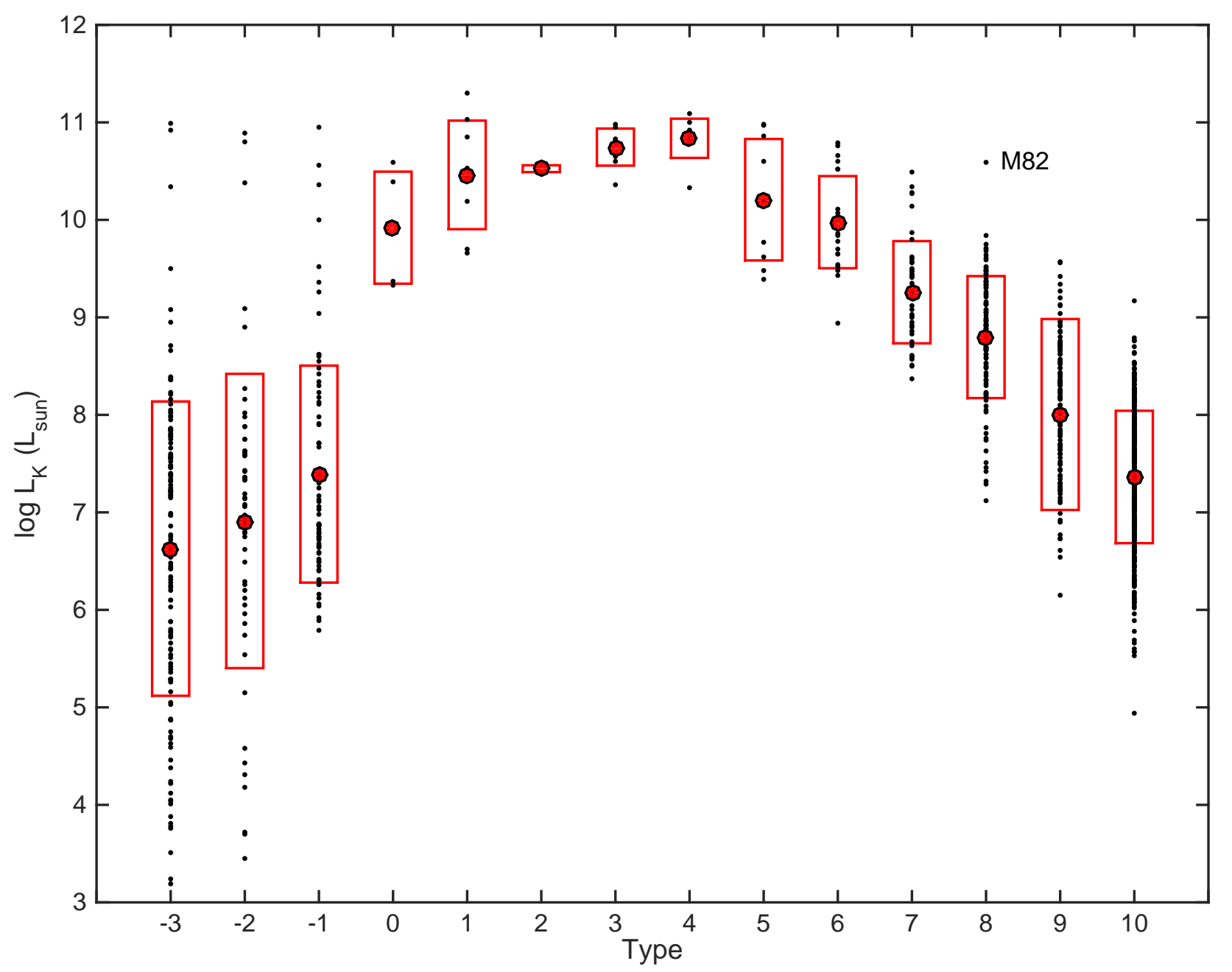

Fig. 2.- Distribution of the LV galaxies on K-band luminosity and morphological type. The mean values of $\log L_{K}$ and standard deviations are marked by large circles and rectangles. 


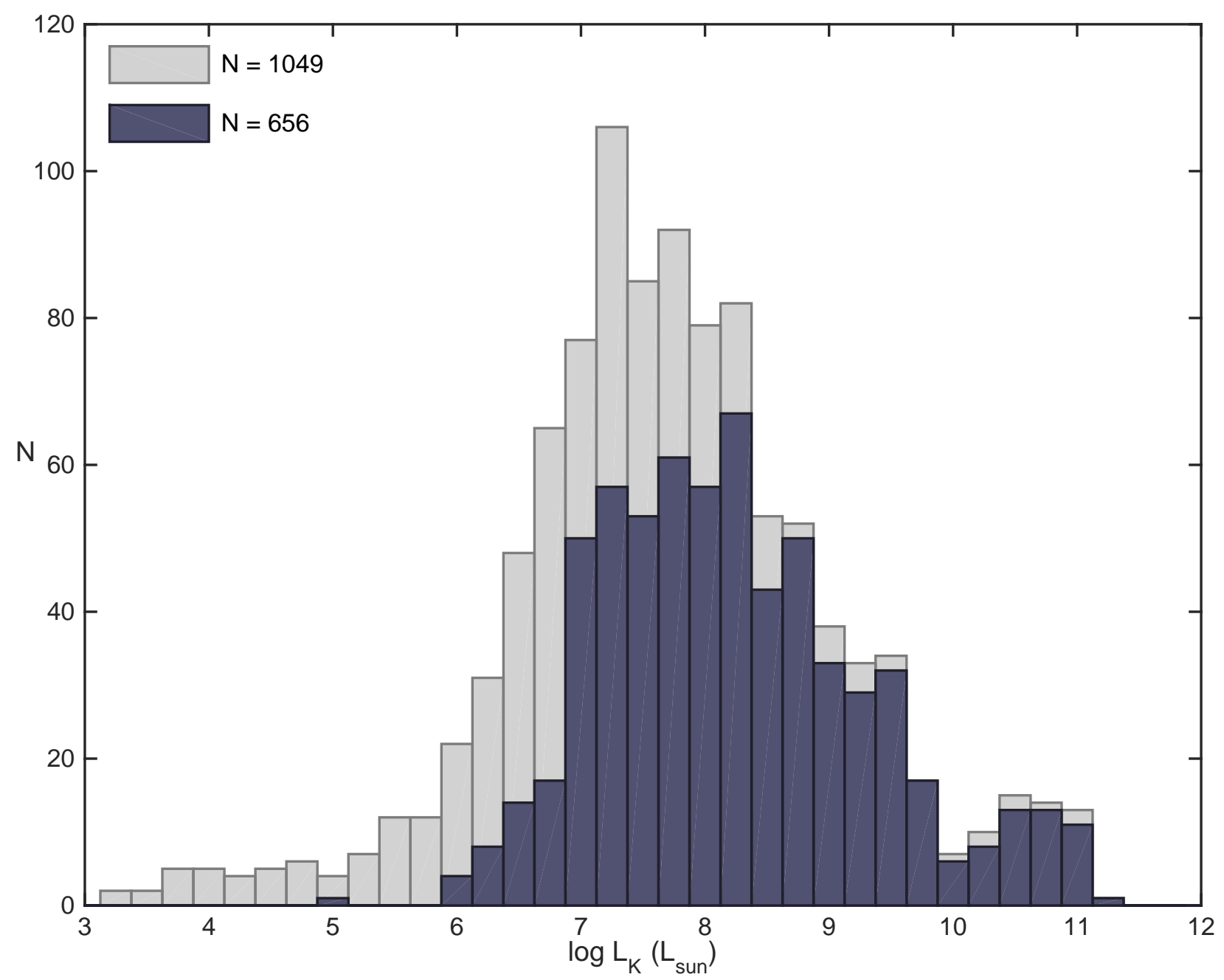

Fig. 3.- Number of the LV galaxies vs. their K-band luminosity. HI-detected galaxies are marked by dark color. 


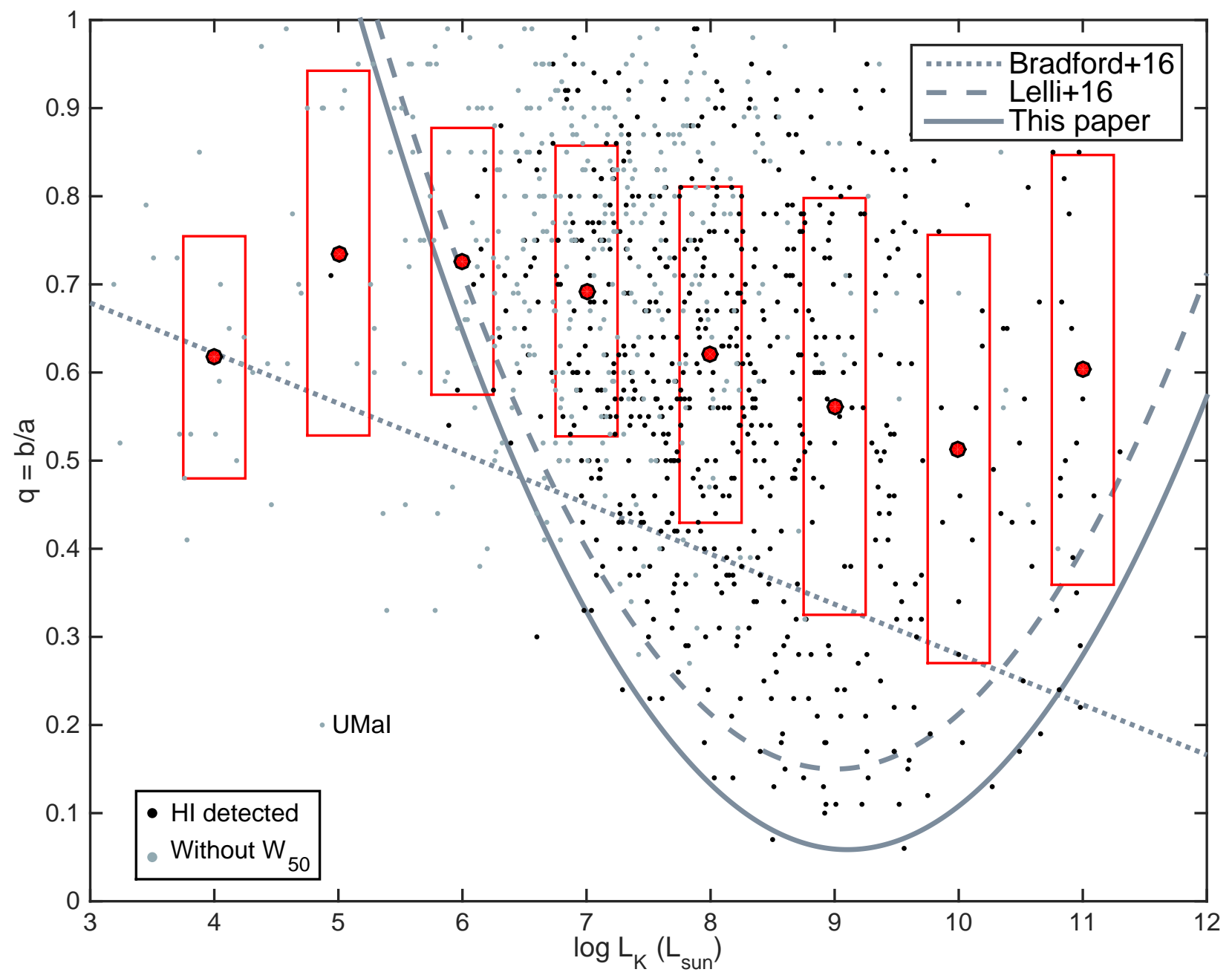

Fig. 4.- Distribution of the LV galaxies on their apparent axial ratio and K-band luminosity. Light and dark small circles indicate HI-detected and non-detected galaxies respectively. The dotted, dashed and solid lines present intrinsic axial ratios under different assumptions. 


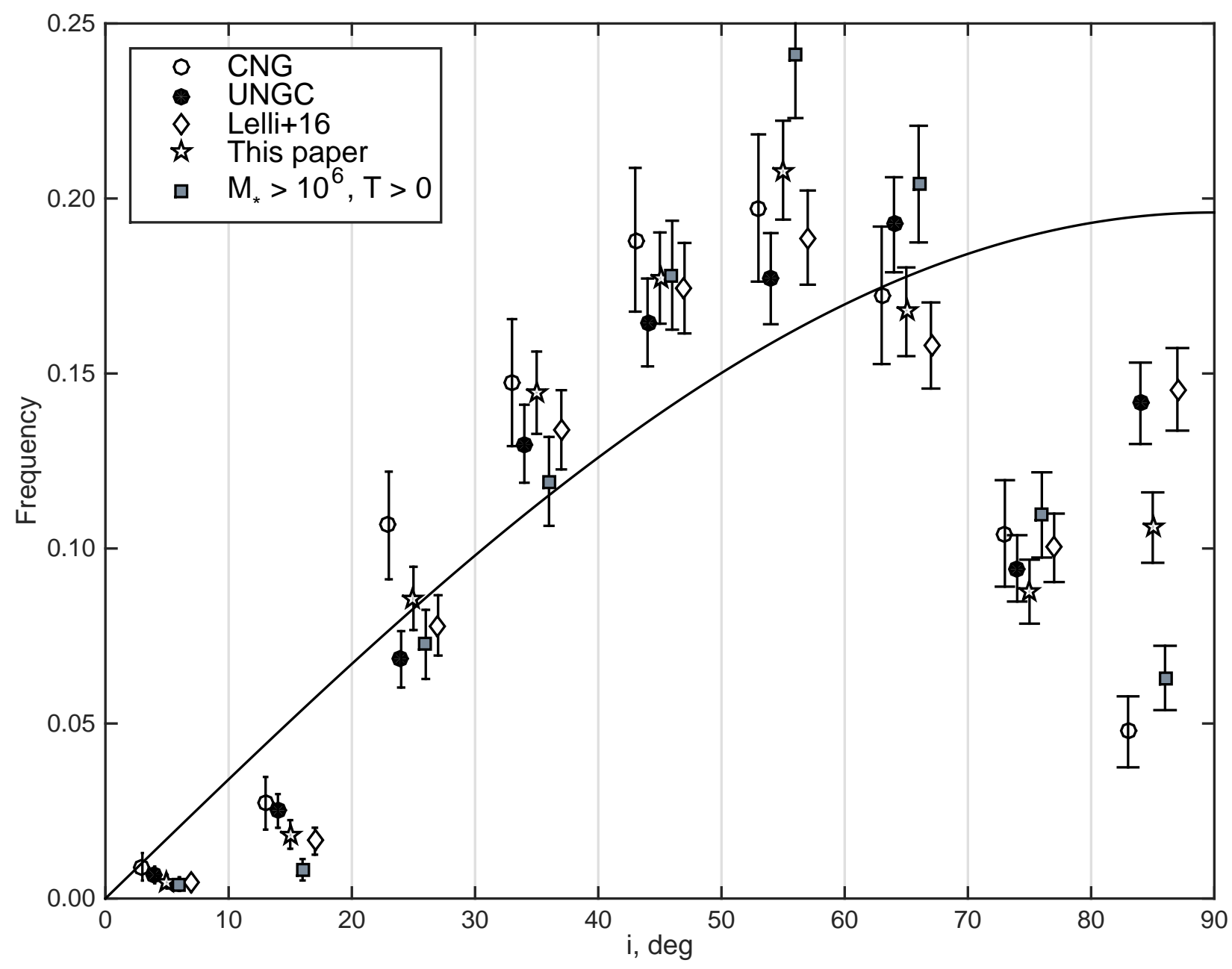

Fig. 5.- The frequency distribution of the LV galaxies on inclination angle $i$ (degree) under different assumptions (2)-(5) about intrinsic axial ratios. The solid line correspondes to the $\sin (i)$ - law expected for arbitrary orientation of galaxy axis. 

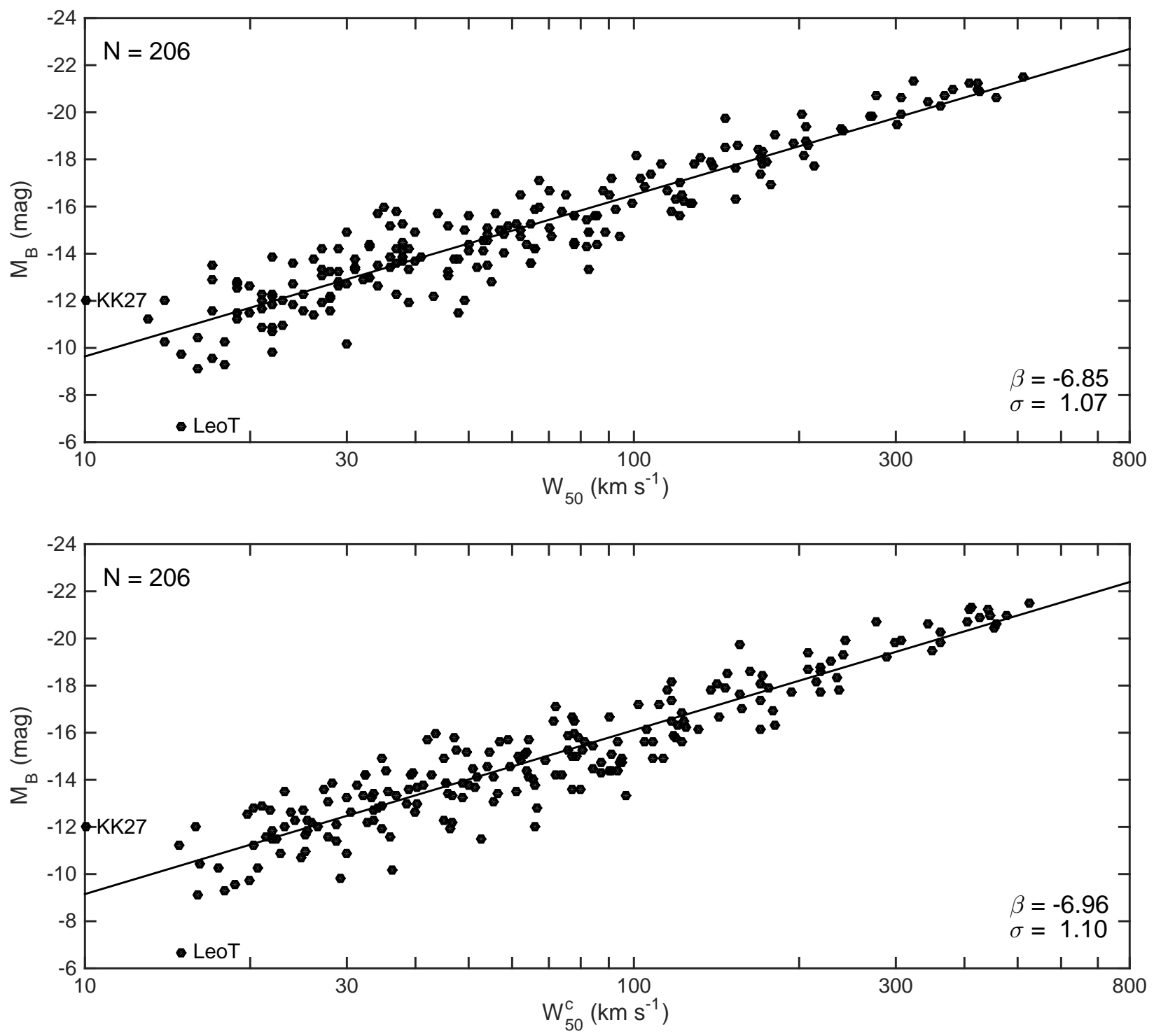

Fig. 6. - B absolute magnitude - line width relation for $206 \mathrm{LV}$ galaxies with accurately measured distances mainly from application of the TRGB and inclinations $i>45^{\circ}$. The absolute magnitudes are corrected for Galactic and internal extinction. Horizontal scales indicate: observed line width $W_{50}$ (upper panel), line width corrected for inclination (bottom panel). The stright lines are leastsquares fits to the ensemble with errors in magnitudes, having a slope $\beta$ and dispersion $\sigma$. 

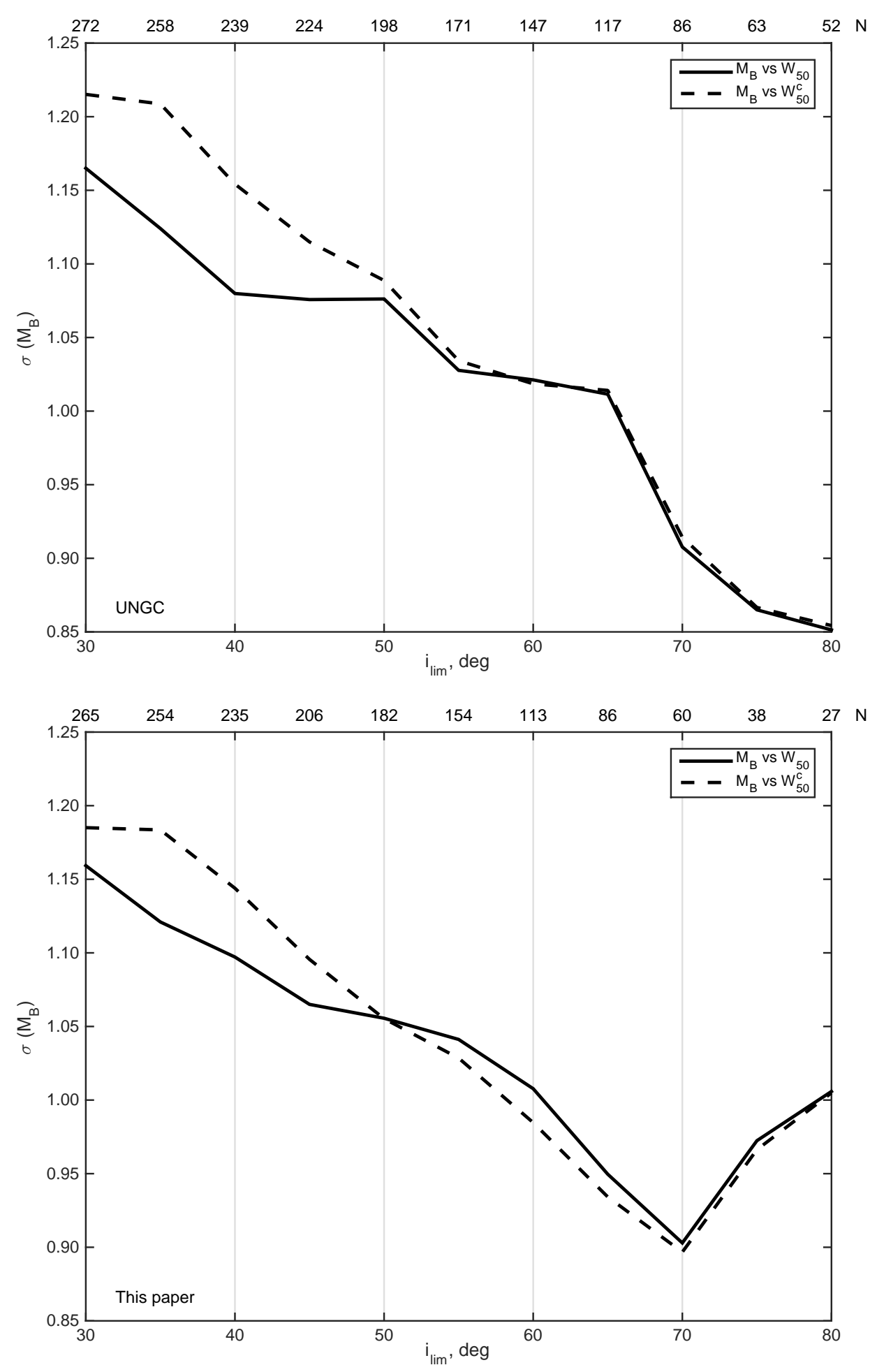

Fig. 7.- Scatter on the TF diagram as a function of limiting inclination angle $i_{\text {lim }}$. The top and bottom panels correspond to the equations (2), and (5), respectively. 

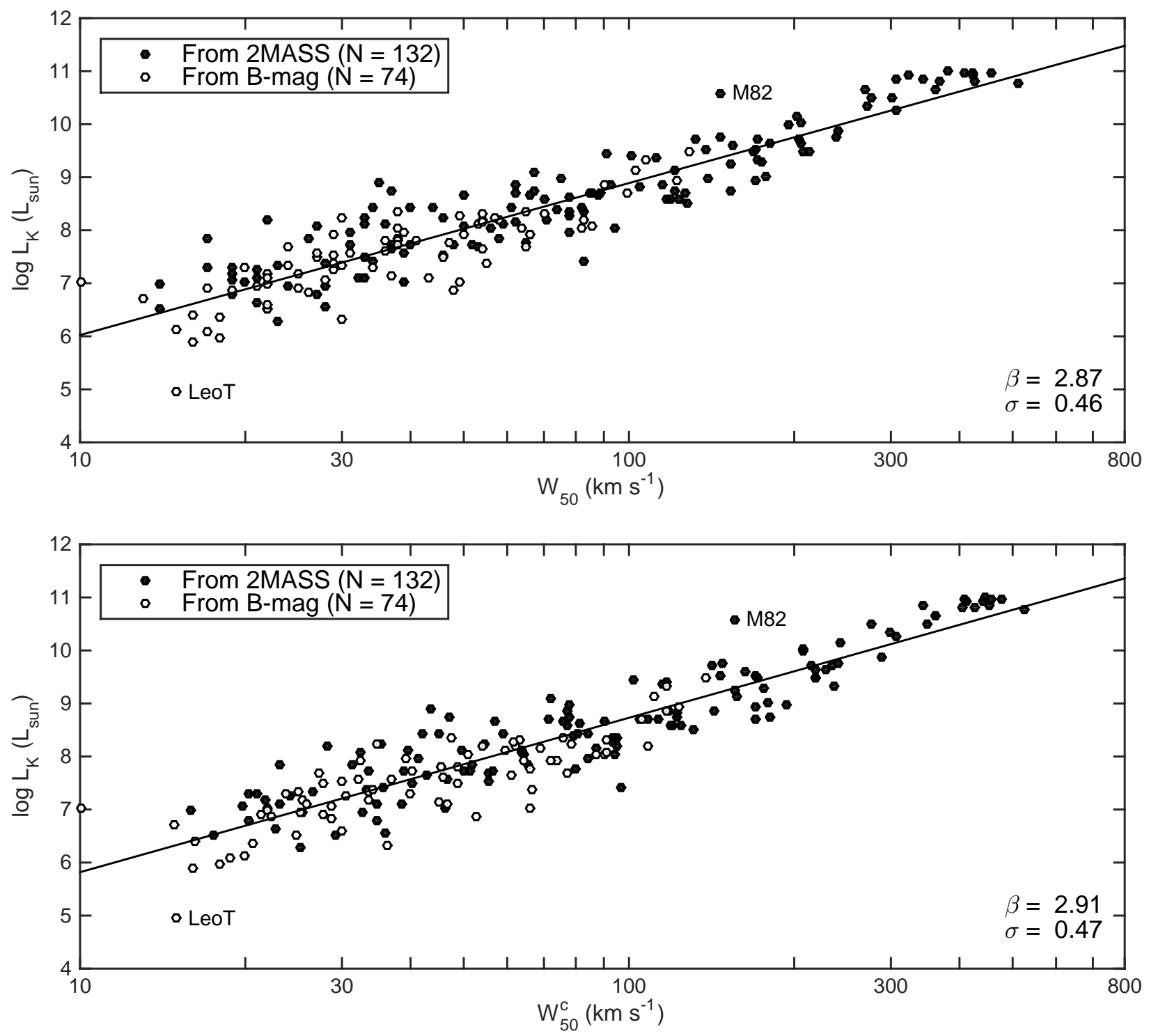

Fig. 8. - $K$-band luminosity versus line width for $206 \mathrm{LV}$ galaxies with accurately determined distances and $i>45^{\circ}$. Upper panel indicates observed line width, bottom panel displays the line width corrected for inclination. Filled circles correspond to 132 galaxies with $K_{s}$ magnitudes from 2MASS and open circles mark 74 galaxies whose $K$ magnitudes are estimated via the mean $B-K$ color and morphological type. Parameters $\beta$ and $\sigma$ in corners indicate the regression slope and dispersion. 

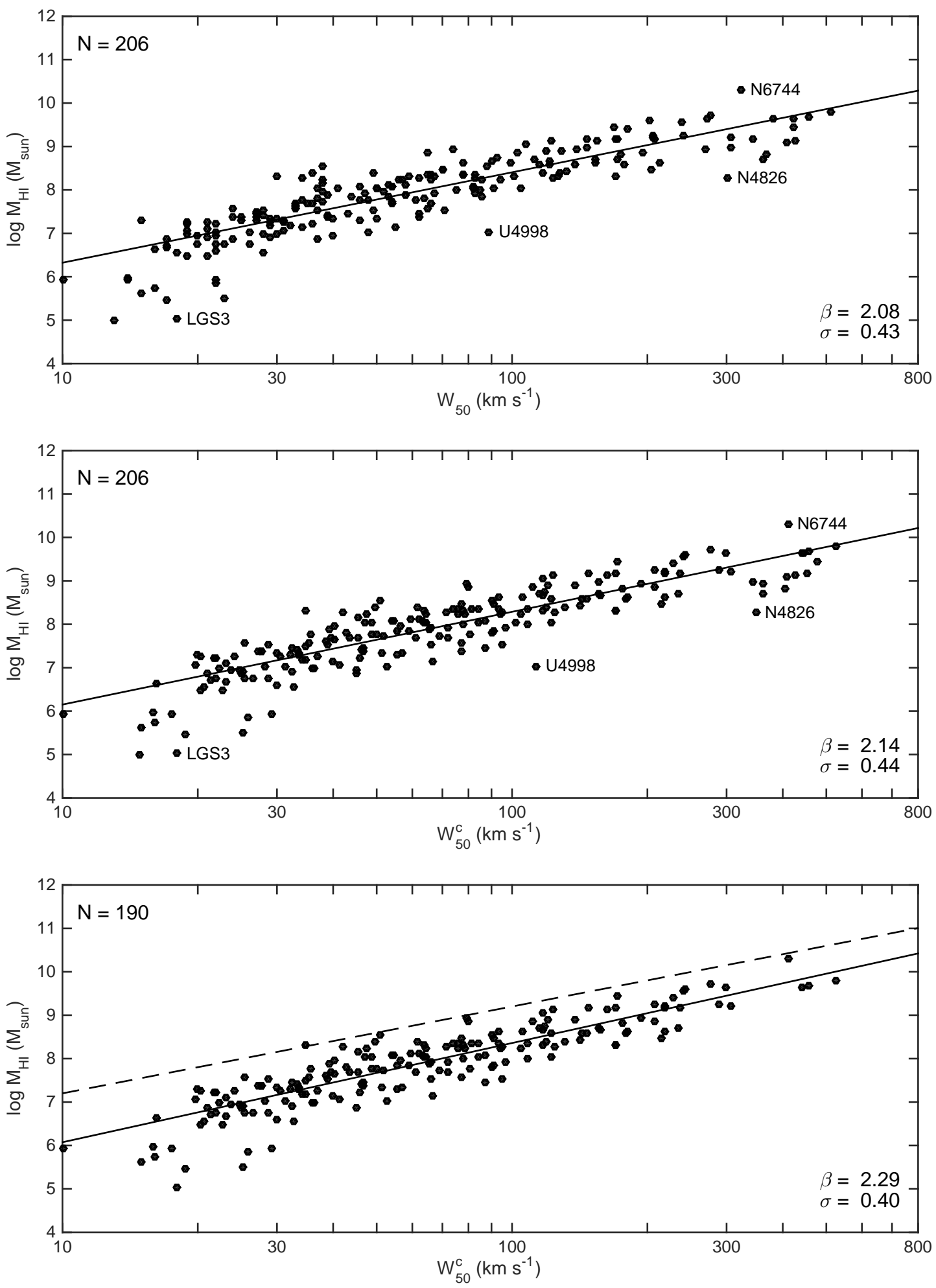

Fig. 9.- The relation between hydrogen mass and line width for LV galaxies with accurately measured distances. Upper and middle panels indicate line width observed and corrected for inclination, respectively. Bottom panel corresponds to 190 gas-rich galaxies with $m_{21}-K<6.0^{m}$. The dashed line indicates the upper limit for the ratio $M_{H I} / W_{50}^{2}$ interpreted as a threshould of gravitation instability favouring star formation. 

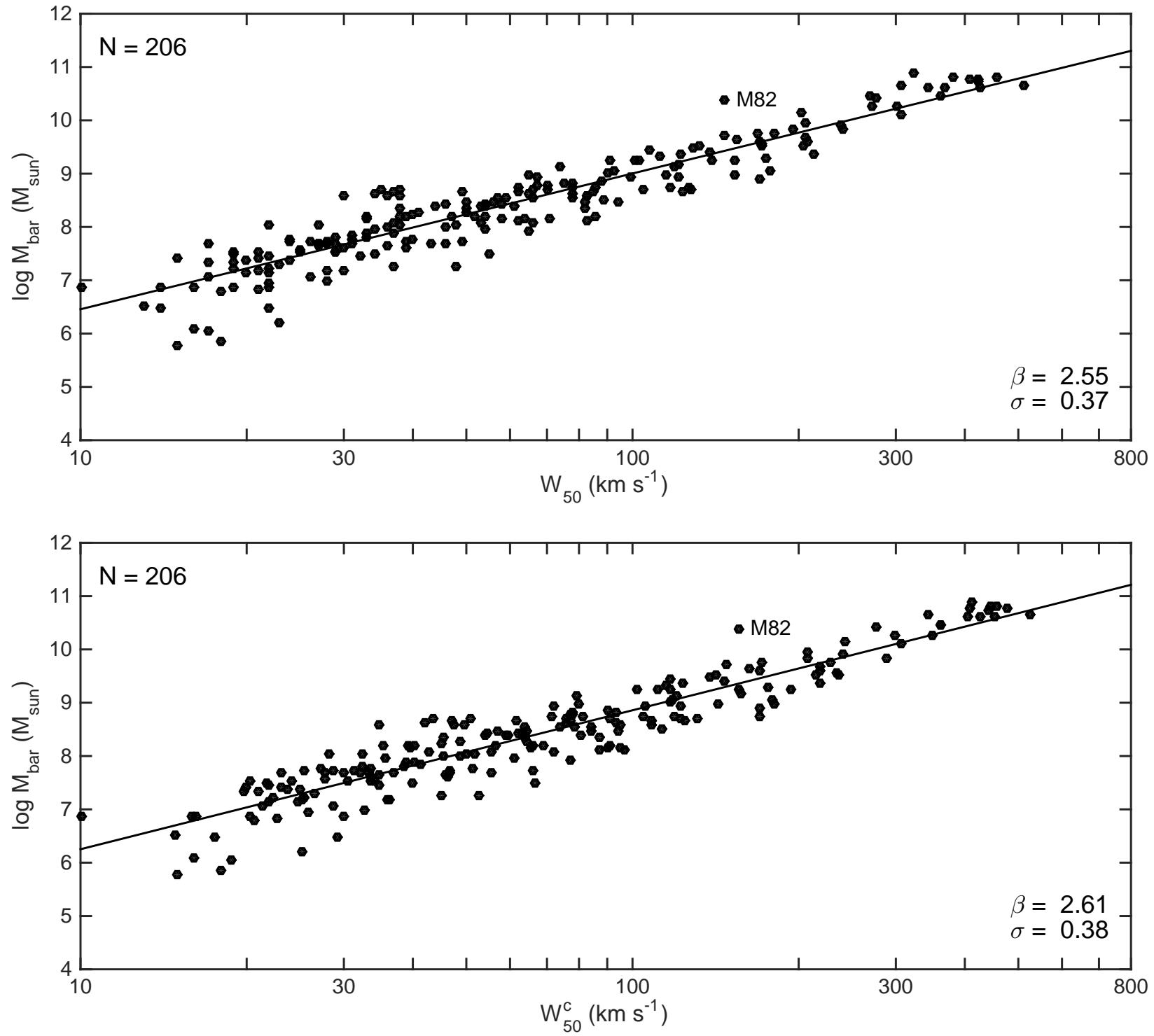

Fig. 10.- Baryonic mass versus observed (upper panel) and corrected for inclination (bottom panel) line width for $206 \mathrm{LV}$ galaxies with accurate distances. The adopted value of stellar massto-luminosity ratio is $\Upsilon^{*}=0.60$ and gaseous mass-to-HI-mass ratio $\eta=1.33$. 


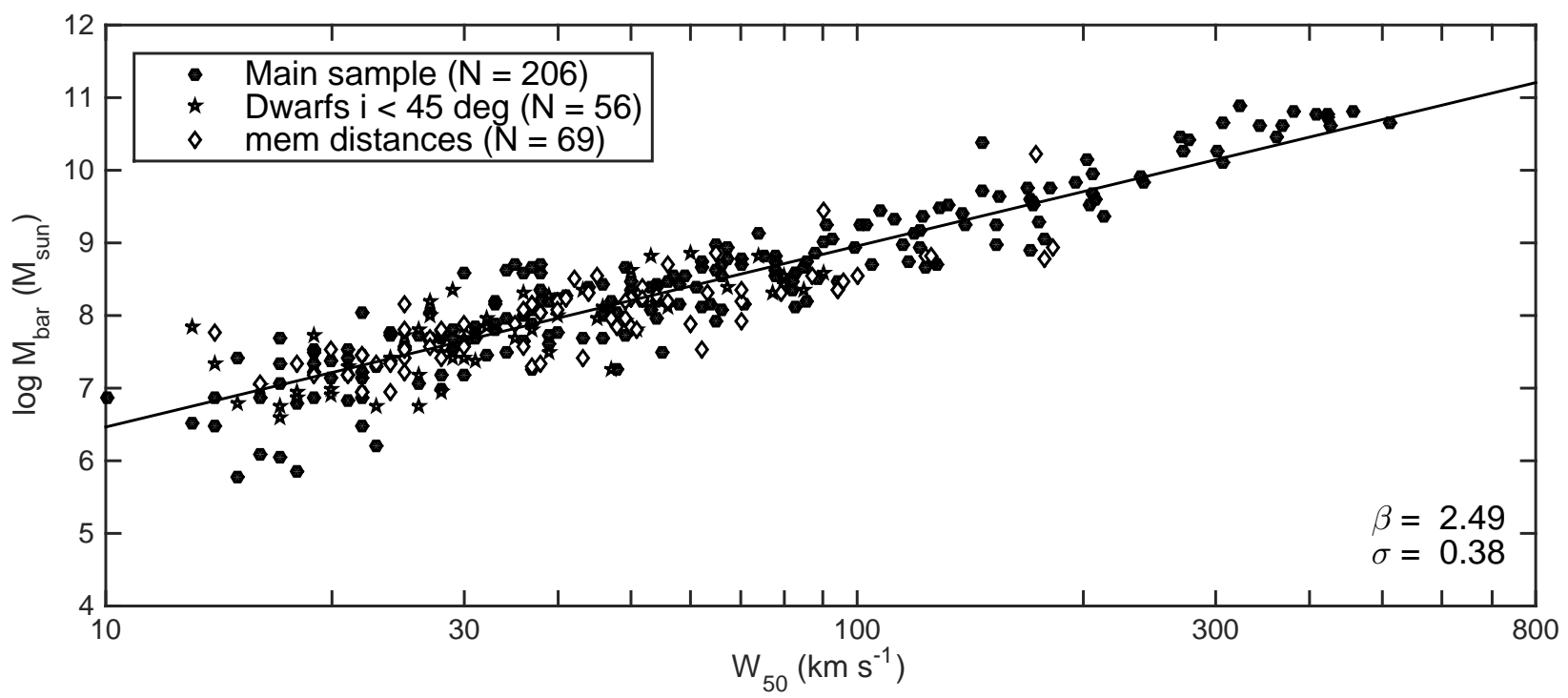

Fig. 11.- The baryonic TF relation for $206 \mathrm{LV}$ galaxies with accurately measured distances (filled circles), 56 low luminosity dwarfs with $i<45^{\circ}$ (crosses) and 69 dwarfs with distances determined via membership in the known groups (diamonds). 

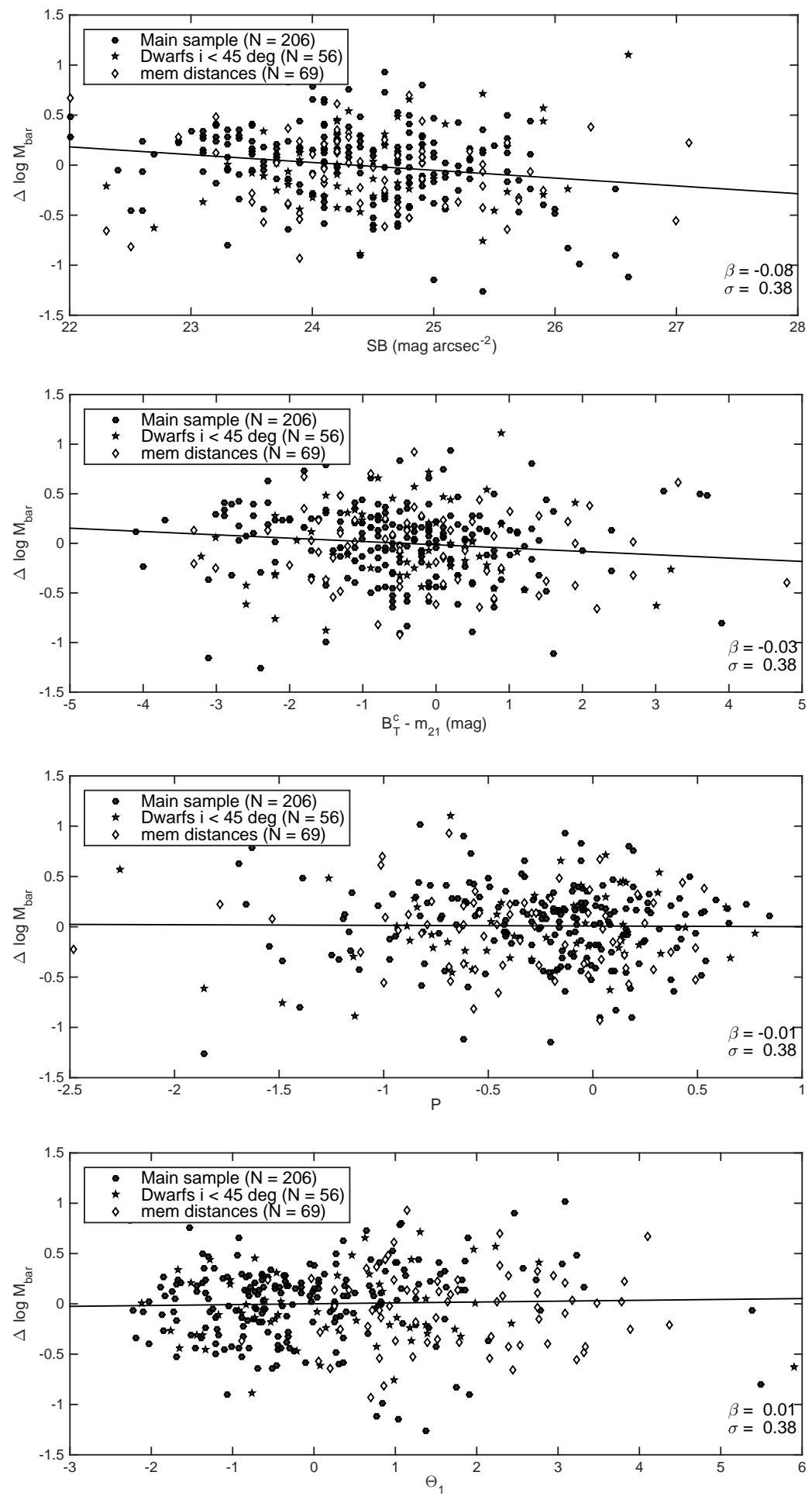

Fig. 12.- Residuals in the baryonic TF diagram as a function of the galaxy surface brightness (upper panel), $\left(B-m_{21}\right)$ gas fraction index (upper middle panel), specific star formation rate expressed in the age of Universe (lower middle panel) and isolation index (bottom panel). Designation of galaxy sub-samples is the same as in Fig.11. The stright lines display regression lines with corresponding slope $\beta$ and scatter $\sigma$. 


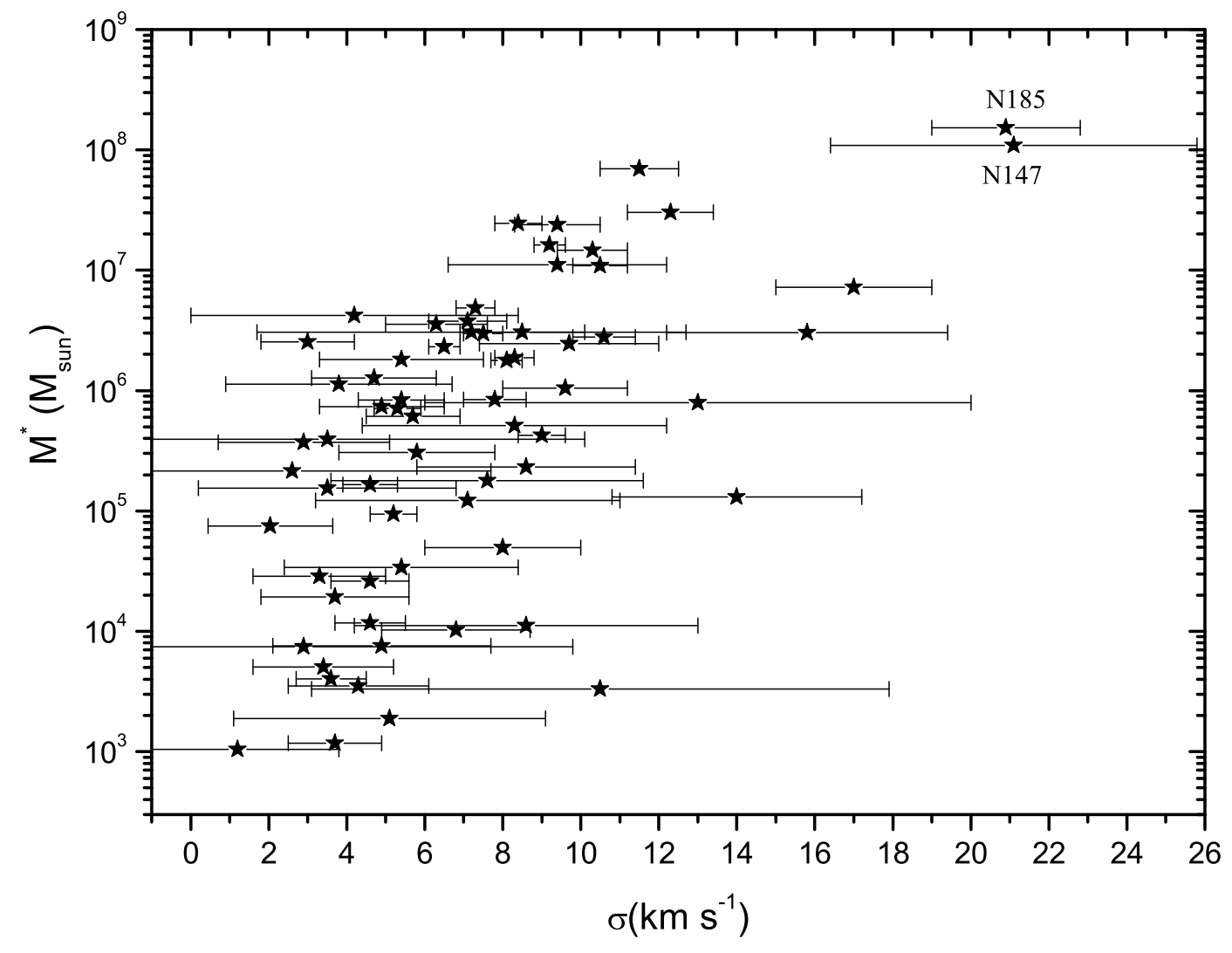

Fig. 13.- Stellar mass versus stellar radial velocity dispersion for $69 \mathrm{dSph}$ and $2 \mathrm{dE}$ galaxies around the Milky Way and M31. Horisontal bars indicate measurement errors. 


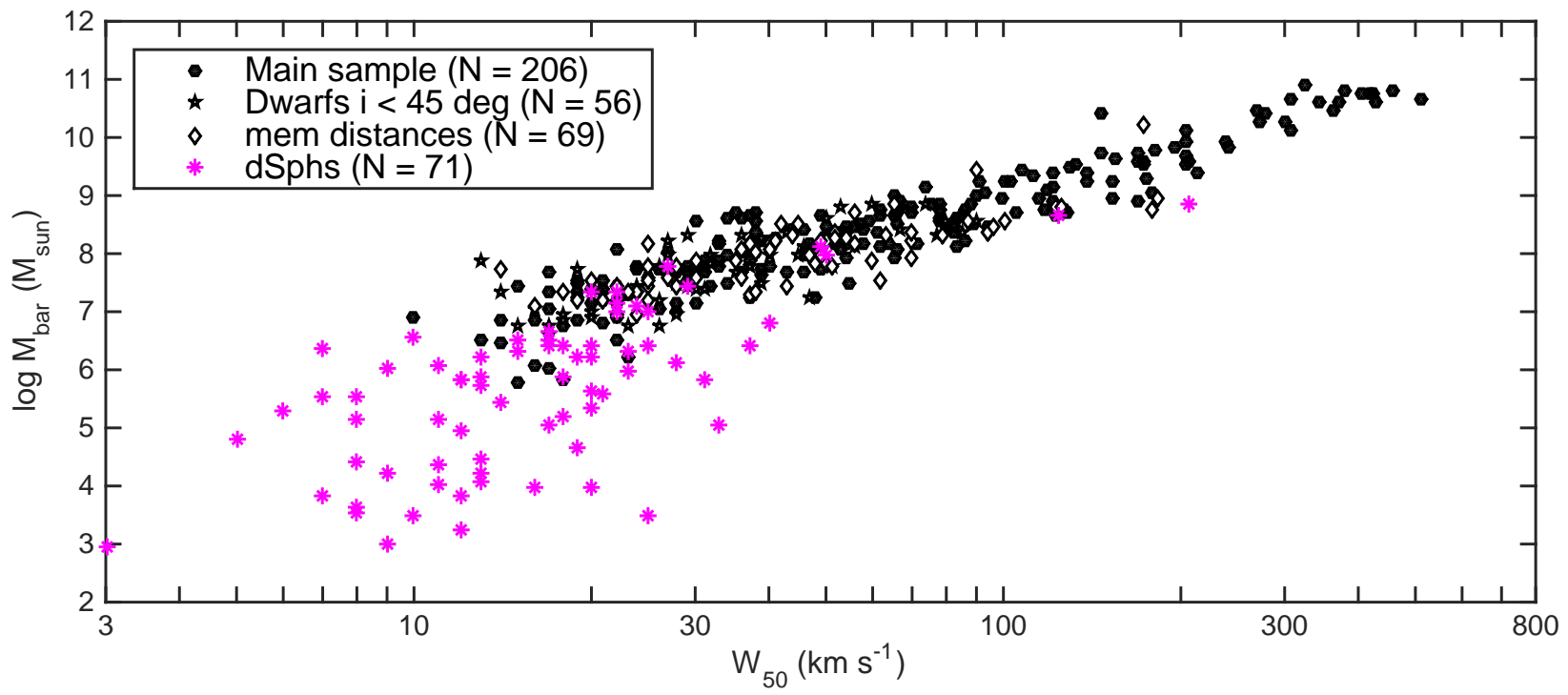

Fig. 14. - The baryonic TF relation for 402 LV galaxies. The dSph companions of the Milky Way and M31 are indicated by red stars. 
Table 5: The initial data for the Local Volume galaxies. Table 5 is published in its entirety in the machine-readable format. A portion is shown here for guidance regarding its form and content

\begin{tabular}{lcrllllrr}
\hline name & $\mathrm{j} 2000$ & $\mathrm{~T}$ & $\mathrm{~b} / \mathrm{a}$ & $W_{50}$ & $\mathrm{D}$ method & $\log \left(L_{K}\right)$ & $\log M_{H I}$ & $M_{\text {bar }}$ \\
\hline$(1)$ & $(2)$ & $(3)$ & $(4)$ & $(5)$ & $(6)$ & $(7)$ & $(8)$ & $(9)$ \\
\hline WLM & $000158.1-152740$ & 9 & 0.35 & 53 & 0.98 TRGB & 7.70 & 7.84 & 8.09 \\
And XVIII & $000214.5+450520$ & -3 & 0.99 & $23^{*}$ & 1.31 TRGB & 6.56 & 6.65 & 6.34 \\
ESO409-015 & $000531.8-280553$ & 9 & 0.46 & 53 & 8.71 TRGB & 8.10 & 8.10 & 8.39 \\
AGC748778 & $000634.4+153039$ & 10 & 0.52 & 16 & 6.22 TRGB & 6.39 & 6.64 & 6.86 \\
And XX & $000730.7+350756$ & -3 & 0.70 & $17^{*}$ & 0.80 TRGB & 5.26 & & 5.04 \\
\hline
\end{tabular}

